Multistep Generalized Transformation Method Applied to Solving Equations of Discrete and Continuous Time-Fractional Enzyme Kinetics

Z. Vosika, V.V. Mitić, A. Vasić, G. Lazović, L. Matija, Lj.M. Kocić

PII:

DOI:

Reference:

To appear in:

Received date:

Revised date:

Accepted date:
S1007-5704(16)30308-2

10.1016/j.cnsns.2016.08.024

CNSNS 3973
Communications in

Nonlinear Science and

Numerical Simulation

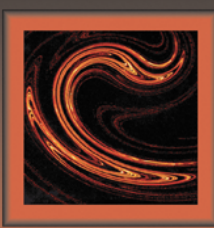

Please cite this article as: Z. Vosika, V.V. Mitić, A. Vasić, G. Lazović, L. Matija, Lj.M. Kocić, Multistep Generalized Transformation Method Applied to Solving Equations of Discrete and Continuous TimeFractional Enzyme Kinetics, Communications in Nonlinear Science and Numerical Simulation (2016), doi: 10.1016/j.cnsns.2016.08.024

This is a PDF file of an unedited manuscript that has been accepted for publication. As a service to our customers we are providing this early version of the manuscript. The manuscript will undergo copyediting, typesetting, and review of the resulting proof before it is published in its final form. Please note that during the production process errors may be discovered which could affect the content, and all legal disclaimers that apply to the journal pertain. 


\section{Highlights}

- New model of Michaelis-Menten kinetic reaction is proposed and investigated.

- New fractional difference-differential calculus Caputo type is introduced.

- Multi-step generalized difference transformation method is proposed and presented.

- Discussed the assumptions under which mentioned fractional discrete time model is valid.

- Discussed conditions for its further development and experimental verification are described. 


\title{
Multistep Generalized Transformation Method Applied to Solving Equations of Discrete and Continuous Time-Fractional Enzyme Kinetics
}

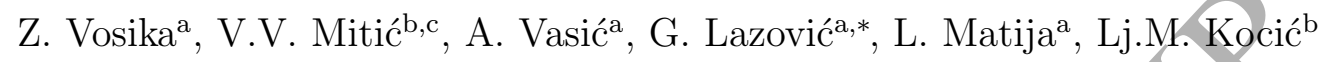 \\ ${ }^{a}$ Faculty of Mechanical Engineering, University of Belgrade, Kraljice Marije 16. \\ Belgrade, 11120/35, Serbia \\ ${ }^{b}$ University of Niš, Faculty of Electronic Engineering, Aleksandra Medvedeva 14, Niš, \\ Serbia \\ ${ }^{c}$ Institute of Technical Sciences of SASA, Belgrade, Serbia
}

\begin{abstract}
In this paper, Caputo based Michaelis - Menten kinetic model based on Time Scale Calculus (TSC) is proposed. The main reason for its consideration is a study of tumor cells population growth dynamics. In the particular case discrete-continuous time kinetics, Michaelis-Menten model is numerically treated, using a new algorithm proposed by authors, called multistep generalized difference transformation method (MSGDETM). In addition numerical simulations are performed and is shown that it represents the upgrade of the multi-step variant of generalized differential transformation method (MSGDTM). A possible conditions for its further development are discussed and possible experimental verification is described.
\end{abstract}

\section{Introduction}

Complex processes in physical, biological, biochemical and similar systems constitute a field of different challenging problems for professional researchers. In most of the cases, the approach is based on the use of the system of a time-delayed integro-differential equations or partial differential equations $[1],[2],[3],[4],[5]$. More recent mathematical and computational framework for analyzing and simulating this system is based on TSC [6], [7], [8].

\footnotetext{
${ }^{*}$ Corresponding author

Email address: glazovic@mas.bg.ac.rs (G. Lazović)
} 
This calculus has three important advantages: (1) more degrees of freedom in the model considered, (2) a "memory effect" that can be included in the model and (3) inherent scaling properties. A discrete or continuous fractional calculus provides a useful mathematical tool for the description of various dynamic phenomena mentioned above. The Michaelis-Menten mechanism is one of the best known and simplest biochemical network (for more details on the enzyme dynamics, please refer to references [2], [9]-[14] and the references therein). Corresponding model of enzyme kinetics is based on the concept of an enzyme, reversibly forming a non-covalent intermediate complex with its substrate before catalyzing the reaction, and then irreversibly dissociating from the product. In that case, enzyme reaction model is given by

$$
E+S \underset{k_{-1}}{\stackrel{k_{+1}}{\rightleftharpoons}} E S \rightarrow E P \stackrel{k_{2}}{\rightarrow} E+P
$$

where $E$ is the enzyme, $S$ the substrate, $E S$ and $E P$ are the enzymesubstrate and enzyme-product intermediate complex, $P$ is a product [2]. The parameters $k_{1}, k_{-1}$, and $k_{2}$ are positive rate constants. If the concentration of the reactants is denoted by lower case letters: $e=[E], s=[S], c=[E S]$, $p=[P]$ and the law of mass action is applied, the time evolution of scheme (1) can be determined from the solution of the system of coupled nonlinear ordinary differential equations (ODEs) [11], [12],[14]:

$$
\begin{array}{r}
\frac{d s}{d t}=-k_{1} e s+k_{-1} c \\
\frac{d e}{d t}=-k_{1} e s+\left(k_{-1}+k_{2}\right) c \\
\frac{d c}{d t}=k_{1} e s-\left(k_{-1}+k_{2}\right) c \\
\frac{d p}{d t}=k_{2} c
\end{array}
$$

subject to the initial conditions

$$
e(0)=e_{0}, s(0)=s_{0}, c(0)=0, p(0)=0 .
$$

In general, the assumption of irreversibility of the Michaelis-Menten reaction (i.e. $k_{-2}=0$ ) is acceptable under following conditions [2]: (1) the 
Gibbs free energy released in the reaction is very large and (2) the substrate concentration is much higher than that of product. In other words, it is valid under standard in vitro assay conditions, and for many in vivo biological reactions, particularly where the product is continually removed by a subsequent reaction. In cases where none of these two conditions apply, the Michaelis-Menten reaction cannot be presented by previous equations. Instead more complex modelling approaches which explicitly take forward and reverse reactions into account, must be adopted to understand the enzyme kinetics.

System (2) can be reduced to three equations for $s, c$ and $p$. By introducing the following parameters and functions:

$$
\begin{array}{r}
\tau=\frac{k_{1} e_{0} t}{\varepsilon}, u(\tau)=\frac{s(t)}{s_{0}}, \nu(\tau)=\frac{c(t)}{e_{0}}, \\
w(\tau)=\frac{p(t)}{e_{0}}, \lambda=\frac{k_{2}}{k_{1} s_{0}}, k_{3}=\frac{k_{-1}+k_{2}}{k_{1} s_{0}}, \varepsilon=\frac{e_{0}}{s_{0}},
\end{array}
$$

reduced system of equations (19) and the initial conditions (3) can be represented in dimensionless form as follows :

$$
\begin{array}{r}
\frac{d u}{d t}=-\varepsilon u+\varepsilon\left(u+k_{3}-\lambda\right) v, \\
\frac{d v}{d t}=u-\left(u+k_{3}\right) v, \\
\frac{d w}{d t}=\lambda v, \\
u(0)=1, v(0)=0, w(0)=0
\end{array}
$$

where $\lambda, k_{3}$, and $\varepsilon$ are dimensionless parameters. The author in reference [14] suggests that in the equations (5) instead of ordinary, it is better to use appropriate Caputo fractional time derivatives: $\frac{d u}{d t} \rightarrow\left({ }_{0}^{C} D_{t}^{\alpha_{1}}\right) u, \frac{d v}{d t} \rightarrow$ $\left({ }_{0}^{C} D_{t}^{\alpha_{2}}\right) v$ and $\frac{d w}{d t} \rightarrow\left({ }_{0}^{C} D_{t}^{\alpha_{3}}\right) w$, where $0<\alpha_{i} \leq 1$ for $i=1,2,3$ are parameters that describe the order of the fractional time-derivatives from the Caputo point of view.

Generalized differential transformation method (GDTM) [15],[16],[17],[18], is one of the semi-numerical analytical methods for fractional, ordinary and partial differential equations that uses the form of generalized Taylor's poly- 
nomials as approximations of the exact solutions. The approximate solutions obtained by using GDTM are valid only in a short time period, while the ones obtained by using the MSGDTM [14], [19], [20] are valid for wider time intervals. MSGDTM may be treated as an algorithm in a sequence of small intervals (i.e., time steps $\Delta t$ ) for finding accurate approximate solutions of the corresponding systems. This method is one of the tools for solving different classes of nonlinear problems.

The following text presents a basic review of the MSGDTM (GDTM), starting with the definition of MSDTM. The ordinary differential transformation of the $k$-th derivative of time function $f(t)$ is defined as follows [14], [18] $\left(\alpha_{i}=1\right)$ :

$$
F(k)=\frac{1}{k !}\left[\frac{d^{k} f(t)}{d t^{k}}\right]_{t=t_{0}}
$$

where $f(t)$ is the original function and $F(k)$ is the transformed function (Taylor coefficients). The differential inverse transformation of $F(k)$ is defined as a Taylor series:

$$
f(t)=\sum_{k=0}^{\infty} F(k)\left(t-t_{0}\right)^{k} .
$$

For implementation purposes, the function $f(t)$ is expressed by a finite series, and (7) can be written as

$$
f(t) \approx \sum_{k=0}^{K} F(k)\left(t-t_{0}\right)^{k}
$$

In this respect, we consider the following nonlinear initial value problem,

$$
\frac{d f}{d t}=G(t, f)
$$

subject to the initial condition $f(0)=c_{0} ; G(t, f)$ is a real function.

Let $[0, T]$ be the interval over which we want to find the solution of the initial value problem (9). The multi-step differential transformation method (MSDTM) assumes that the interval $[0, T]$ is divided into M subintervals $\left[t_{m-1}, t_{m}\right], m=1,2, \ldots, M$ of equal step size $\Delta t=T / M$ by using the nodes $t_{m}=m \Delta t, t_{0}=0$. A sequence of approximate solutions $f_{m}(t)$, 
$m=1,2, \ldots, M$, for the solution $f(t)$ expressed by

$$
f(t)= \begin{cases}f_{1}(t), & t \in\left[0, t_{1}\right], \\ f_{2}(t), & t \in\left[t_{1}, t_{2}\right], \\ \vdots & t \in\left[t_{M-1}, t_{M}\right],\end{cases}
$$

taking into account (6) and (8)

$$
f_{m}(t)=\sum_{n=0}^{N} F(n, m)\left(t-t_{m-1}\right)^{n},
$$

with the initial conditions: for $k=2,3, \ldots, M, f_{k}\left(t_{k-1}\right) \equiv F(0, k)=$ $\sum_{n=0}^{N} F(n, k-1)\left(t_{k-1}-t_{k-2}\right)^{n}$. The main steps of the MSDTM, are as follow: (1) differential transformation (6) is applied to the problem (9), the result is a recurrence relation; (2) by solving this relation over each time subinterval $\left[t_{m-1}, t_{m}\right], m=1,2, \ldots, M$ and using the differential inverse transformation (7), we can obtain a solution for the problem. If $\Delta t=T$, then MSDTM is reduced to the classical DTM. It is proved [17], [18],[19] that, approximated solutions obtained using DTM are not valid for large $t$ for some systems . MSGDTM considers fractional version of equation (9) in Caputo sense and generalized Taylor series expansion 118], [21]

$$
f(t)=\sum_{k=0}^{\infty} F_{\alpha}(k)\left(t-t_{0}\right)^{k \alpha}, \quad 0<\alpha \leq 1 .
$$

As demonstrated in the paper [19], MSDTM is comparable to RungeKutta fourth order method. MSGDTM considered in references [14], [17], [18], have highly accurate approximate solutions which are applicable over the longer time intervals. The problem of time scale of biological processes is not yet datisfactorily resolved. It is known [6],[7],[8] that, the time scale of many different biological processes may be discrete. In addition, in refference [8], the best observation fit is found for discrete fractional Gompertz curve. Specifically appropriate fractional discrete Caputo operator for modelling time dynamic of tumor growth is used. However, tumor growth models using continuous fractional or ordinary calculus [22], [23], [24], [25], [26], [27], [28] are also known in the literature. The paper [22] used Michaelis-Menten substrate model for the time growth description on the cellular population 
in multicellular organisms. The model in [22] demonstrates that neoplastic cellular proliferation can also be promoted by alterations in the somatic landscape that reduce inhibitory signals produced by the normal cells and extracellular matrix. That is consistent with experimental evidence for a strong micro environmental influence in tumor genesis independent of genomic changes in the neoplastic populations. Within TSC different time scales are possible, but, due to the existence of simple discrete temporal dynamics of some tumors it is possible to assume corresponding MichaelisMenten kinetics. Therefore, the model given in [14] may be discretized in such a way that rather than fractional Caputo derivatives, fractional Caputo differences could be used. To our best knowledge, there are two types of acceptable Caputo differences: (1) a forward Caputo fractional difference described in [29] and (2) a backward Caputo fractional difference published in the papers [30], [31]. The second approach will be generalized in this paper. Other approaches to the problem of establishing a diserete fractional calculus are given in [32] [33].

Various types of kinetics could be described with these operators. These kinetics have basics in appropriate non-extensive thermodynamics, for example, as special case - Tsallis Statistics. One of the latest review of various statistics is presented in [34]. Caputo operator proposed in this article is more powerful for this purpose as generalization of existing one. In this respect, the basic idea behind the research presented here is that it represents the basis for the extraction of discrete fractional Michaelis-Menten model parameters, as is done for model (2) in [35].

This paper is organized as follows. In Section 2, according to [6],[7],[30], [31], the authors provide some basic concepts concerning the fractional nabla calculus in the Caputo sense on time scales, and formulate difference transformation method. In Section 3, basics of MSGDETM is proposed. In Section 4, MSGDETM is applied to the generalized discrete problem in regards to [14]. Numerical results and simulations are presented graphically. The assumptions underlying the model are discussed and conditions for its further development and experimental verification are described. Finally, the conclusions are given in Section 5.

\section{Mathematical preliminaries}

For the concept of continuous fractional derivative, Caputos definition will be adopted and expanded to particular discrete version based on TSC. 
It represents a modification of the Riemann-Liouville definition of derivative and includes correct behavior in relation to the initial conditions. This fact is important for the most of biochemical processes. Taking all this into account, MSGDETM is formulated numerically.

Definition 2.1. ([36]) Let $t \in[a, b] \subset \mathbb{R}$ and function $\phi(t)$ is Lebesgue integrable in $[a, b]: \phi(t) \in L([a, b])$. Then, the left fractional Riemann-Liouville integrals ${ }_{a}^{R L} I_{t}^{\alpha}$ of order $\alpha>0$ are defined by

$$
\left(\begin{array}{l}
R L \\
a
\end{array} T_{t}^{\alpha}\right) \phi(t):=\frac{1}{\Gamma(\alpha)} \int_{a+}^{t}\left(t-t^{\prime}\right)^{\alpha-1} \phi\left(t^{\prime}\right) d t^{\prime}
$$

$\Gamma($.$) is Euler's Gamma function.$

Definition 2.2. The left fractional Caputo derivative of order $0<\alpha \leq 1$. Let $t \in[a, b] \subset \mathbb{R}$ and $\frac{d \phi(t)}{d t} \in L([a, b])$. Then, the left fractional Caputo derivative ${ }_{a}^{C} D_{t}^{\alpha}$ of order $0<\alpha<1$ are defined by

$$
\left({ }_{a}^{C} D_{t}^{\alpha}\right) \phi(t):=\frac{1}{\Gamma(1-\alpha)} \int_{a+}^{t} d t^{\prime}\left(t-t^{\prime}\right)^{-\alpha} \frac{d \phi\left(t^{\prime}\right)}{d t^{\prime}} .
$$

As a complement to the previous definition, for $\alpha=1$, the following relations is valid: $\left({ }_{a}^{C} D_{t}^{1}\right) \phi(t):=\frac{d \phi(t)}{d t}$.

Equation (13) can be rewritten in the form $\left({ }_{a}^{C} D_{t}^{\alpha}\right) \phi(t):=\left({ }_{a}^{R L} I_{t}^{1-\alpha}\right) \frac{d \phi(t)}{d t}$.

In this paper the following definitions, notations and known propositions will be used: $h>0$ and a time scale $\mathbb{T}=h \mathbb{Z}$ put. By $\mathcal{F}_{\mathbb{T}}$ the set of real valued functions defined on $\mathbb{T}: f: \mathbb{T} \rightarrow \mathbb{R}$ will be denoted. For any $t \in \mathbb{T}$, definition of a forward and backward jump operators is: $\sigma(t):=\inf \{s \in \mathbb{T}, s>t\}$, $\rho(t):=\sup \{s \in \mathbb{T}, s<t\}$ and the forward (backward) graininess function $\mu(t):=\sigma(t)-t)(\nu(t):=t-\rho(t))$.

Definition 2.3. ([30], [31]). The left nabla derivative $m$-th degree of the function $f(t)\left(m \in \mathbb{N}_{0}\right)$. Let $t \in \mathbb{T}=h \mathbb{Z}$ (then, $\mu(t)=\nu(t)=h$ ) and $f(t) \in) \mathcal{F}_{\mathbb{T}}$. The first left nabla derivative of $f$ (or, first backward difference of $f$ ), given by

$$
\nabla_{h} f(t):=\frac{f(t)-f(\rho(t))}{\nu(t)} .
$$

If $m=0$, then $\nabla_{h}^{0} f(t):=f(t)$, and, for $m>1$ is $\nabla_{h}^{m} f(t):=\nabla_{h}\left(\nabla_{h}^{m-1} f(t)\right)$. 
Definition 2.4. The left nabla integral (or, first backward sum) of $f \in \mathcal{F}_{\mathbb{T}}$ over the time scale interval $\left[t_{0}, t\right] \subset\left[t_{0}, t_{r}\right],\left[t_{0}, t_{r}\right]:=\left\{t^{\prime} \in \mathbb{T}, t_{0} \leq t^{\prime} \leq t_{r}\right\}$, $t_{0}, t_{r} \in \mathbb{T}$, is defined by

$$
\int_{t_{0}+}^{t} f\left(t^{\prime}\right) \nabla_{h} t^{\prime}:=F(t)-F\left(t_{0}+\right),
$$

where $F$ is an antiderivative of $f$, i.e., the function satisfying $\nabla_{h} F^{\prime}=$ $f$ on $\left[t_{0}, t_{r}\right]$ (or, otherwise written, $\left(t_{0}+\nabla_{h}^{-1}\right) f(t)=F(t)-F\left(t_{0}+\right)$ ). If $t_{0}, t_{r} \in \mathbb{T}$ and $t_{0}>t_{r}$, then $\int_{t_{0}+}^{t_{r}} f\left(t^{\prime}\right) \nabla_{h} t^{\prime}=-\int_{b}^{t_{0}+} f\left(t^{\prime}\right) \nabla_{h} t^{\prime}$ and we put $\int_{t_{0}+}^{t_{0}+} f\left(t^{\prime}\right) \nabla_{h} t^{\prime}:=0$. In the form of the sum, the left nabla integral is $\int_{t_{0}+}^{t} f\left(t^{\prime}\right) \nabla_{h} t^{\prime}=$ $\sum_{t^{\prime} \in\left[t_{0}, t\right]} f\left(t^{\prime}\right) \nu\left(t^{\prime}\right)$. By convention, $\int_{t_{0}}^{t} f\left(t^{\prime}\right) \nabla_{h} t^{\prime} \equiv \int_{t_{0}+}^{t} f\left(t^{\prime}\right) \nabla_{h} t^{\prime}\left(t_{0}+\rightarrow t_{0}\right)$.

Definition 2.5. ([37]). For $t, y \in \mathbb{R}$ the $h$-factorial function with regard to $t_{0} \in \mathbb{R}$, on condition $t-t_{0} \geq 0$, is defined by

$$
\left(t-t_{0}\right)_{h}^{(y)}:=h^{y} \frac{\Gamma\left(\frac{t-t_{0}}{h}+1\right)}{\Gamma\left(\frac{t-t_{0}}{h}+1-y\right)}
$$

Its basic features are: 1) $\left.\lim _{h \rightarrow 0+}\left(t-t_{0}\right)_{h}^{(y)}=\left(t-t_{0}\right)^{y} ; 2\right) 0_{h}^{(y)}=\frac{h^{y}}{\Gamma(1-y)}$; 3) for $k \in \mathbb{N}$ and $h>0,0_{h}^{(k)}=0, \theta_{h}^{(0)}=1$; 4) if $0<\alpha \leq 1$ then $0_{h=0+}^{(k \alpha)}=0$ and $0_{h=0+}^{(0)}=1$.

Next lemma is a generalization of the statements from the reference [38]

Lemma 2.1. (i) $\left(\frac{t}{h}-\mu\right) t_{h}^{(\mu)}=t_{h}^{(\mu+1)}$, where $\mu \in \mathbb{R}^{+}$and $h>0$.

(ii) $(\mu h)_{h}^{(\mu)}=h^{\mu} \Gamma(\mu+1)$.
(iii) If $t \leq r$ then $t_{h}^{(\alpha)} \leq r_{h}^{(\alpha)}$ for all $\alpha>r$.
(iv) If $0 \nless \alpha<1$, then $t_{h}^{(\alpha \nu)} \geq\left(t_{h}^{(\nu)}\right)^{\alpha}$.
(v) $t_{h}^{(\alpha+\beta)}=(t-h \beta)_{h}^{(\alpha)} t_{h}^{(\beta)} ; \alpha, \beta \in \mathbb{R}^{+} \cup\{0\}$.

Definition 2.6. ([7]). The discrete and normalized generalized monomials $\hat{h}_{n}: \mathbb{T} \times \mathbb{T} \rightarrow \mathbb{R}, n \in \mathbb{N}_{0}$, is given by

$$
\hat{h}_{n}(t, s)=\left\{\begin{array}{lr}
1, & n=0, \\
\int_{s}^{t} \hat{h}_{n-1}\left(t^{\prime}, s\right) \nabla_{h} t^{\prime}, & n \in \mathbb{N},
\end{array}\right.
$$

for $s, t \in \mathbb{T}$. 
If we let $\nabla_{h} \hat{h}_{n}(t, s)$, for each fixed $s \in \mathbb{T}$, denote the derivatives of $\hat{h}_{n}(t, s)$ with respect to $t$, then $\nabla_{h} \hat{h}_{n}(t, s)=\hat{h}_{n-1}(t, s)$ for $n \in \mathbb{N}, t \in \mathbb{T}$.

Theorem 2.2. Discrete Taylor's formula. Let $n \in \mathbb{N}$ and $t, t_{0} \in \mathbb{T}$. Suppose $f: \mathbb{T} \rightarrow \mathbb{R}$, then

$$
f(t)=\sum_{k=0}^{n} \hat{h}_{k}\left(t, t_{0}\right) \nabla_{h}^{k} f\left(t_{0}\right)+\int_{t_{0}}^{t} \hat{h}_{n}\left(t, \rho\left(t^{\prime}\right)\right) \nabla_{h}^{n+1} f\left(t^{\prime}\right) \nabla_{h} t^{t}
$$

An important relation between the generalized exponential function and the monomials is given by

$$
\hat{e}_{\lambda}(t, s)=\sum_{k=0}^{\infty} \lambda^{k} \hat{h}_{k}(t, s) \quad s, t \in \mathbb{T} ; t \geq s,
$$

where $\lambda \in \mathcal{R}_{c}(\mathbb{T})[31]$.

The normalized extension of the discrete monomials $\hat{h}_{n}(t, s)$ takes the form

$$
\hat{h}_{\alpha}(t, s):=\frac{(t-s)_{h}^{(\alpha)}}{\Gamma(\alpha+1)}, \alpha \in \mathbb{R} .
$$

For $t_{0} \in \mathbb{T}$, the following restricted $h$-time scale was considered:

$$
\tilde{\mathbb{T}}^{\sigma^{i}\left(t_{0}\right)}: \hat{=}\left\{t \in \mathbb{P}, t \geq \sigma^{i}\left(t_{0}\right)\right\} \quad i=0,1,2, \ldots,
$$

where the symbol $\sigma^{i}$ stands for the $i$ th iterate of $\sigma, \sigma^{0}\left(t_{0}\right)=t_{0}$ (analogously, we use the symbol $\rho^{i}$ ).

Lemma 2.3. Let $n, m \in \mathbb{N}, n \geq m$ be such that $t=\sigma^{n}(s), \alpha \in \mathbb{R}, s, t \in \mathbb{T}$. Then

$$
\nabla_{h}^{m} \hat{h}_{\alpha}(t, s)=\left\{\begin{array}{lr}
\hat{h}_{\alpha-m}(t, s), & \alpha \notin\{0,1, \ldots, m-1\}, \\
0, & \alpha \in\{0,1, \ldots, m-1\} .
\end{array}\right.
$$

Definition 2.7. The left nabla $h$-fractional integral (in the sense of RiemannLiouville) of order $\alpha \in \mathbb{R}^{+}$over the time scale interval $\left[t_{0}, t\right] \cap \tilde{\mathbb{T}}^{t_{0}}$ is defined by

$$
\left(\begin{array}{l}
R L \\
t_{0}
\end{array} \nabla_{h}^{-\alpha}\right) f(t):=\int_{t_{0}}^{t} \hat{h}_{\alpha-1}\left(t, \rho\left(t^{\prime}\right)\right) f\left(t^{\prime}\right) \nabla_{h} t^{\prime} .
$$


Mentioned operator $\left(\begin{array}{l}R L \\ t_{0}\end{array} \nabla_{h}^{-\alpha}\right)$, as the first operator in this calculus, is a linear function ${ }_{t_{0}}^{R L} \nabla_{h}^{-\alpha}: \mathcal{F}_{\mathbb{T}} \rightarrow \mathcal{F}_{\mathbb{T}}$, i.e., over the same time scale. Analogous operator in the reference [29] changes the time scale. This is the main reason why the results of work presented in [31] is used.

Theorem 2.4. Let $\alpha, \beta \in \mathbb{R}^{+},\left[t_{0}, t\right] \cap \tilde{\mathbb{T}}^{t_{0}}$, follow relations are valid

$$
\left(\left(\begin{array}{l}
R L \\
t_{0}
\end{array} \nabla_{h}^{-\alpha}\right)\left(\begin{array}{l}
R L \\
t_{0}
\end{array} \nabla_{h}^{-\beta}\right)\right) f(t)=\left({ }_{t_{0}}^{R L} \nabla_{h}^{-(\alpha+\beta)}\right) f(t) .
$$

Definition 2.8. The left nabla h-fractional derivative (in the sense of $C a$ puto) of order $\alpha \in(0,1]$ over the time scale interval $\left[t_{0}, t\right] \cap \tilde{\mathbb{T}}^{t_{0}}$ is defined by

$$
\left(\begin{array}{l}
C \\
t_{0}
\end{array} \nabla_{h}^{\alpha}\right) f(t):=\left({ }_{t_{0}}^{R L} \nabla_{h}^{-(1-\alpha)}\right) \nabla_{h} f(t)\left(=\int_{t_{0}}^{t} \nabla_{h} t^{\prime} \hat{h}_{-\alpha}\left(t, \rho\left(t^{\prime}\right)\right) \nabla_{h} f\left(t^{\prime}\right)\right) .
$$

Theorem 2.5. If $\alpha \in(0,1],\left[t_{0}, t\right] \cap \tilde{\mathbb{T}}^{t_{0}}$, follow relations are valid

$$
\begin{aligned}
& \left(\left(\begin{array}{c}
C \\
t_{0}
\end{array} \nabla_{h}^{\alpha}\right)\left(\begin{array}{l}
R L \\
t_{0}
\end{array} \nabla_{h}^{-\alpha}\right)\right) f(t)=f(t), \\
& \quad\left(\left(\begin{array}{l}
R L \\
t_{0}
\end{array} \nabla_{h}^{-\alpha}\right)\left(\begin{array}{l}
C \\
t_{0}
\end{array} \nabla_{h}^{\alpha}\right)\right) f(t)=f(t)-f\left(t_{0}\right) .
\end{aligned}
$$

The following two lemmas are relevant.

Lemma 2.6. [39]. Let $\alpha \in \mathbb{R}^{+}, \beta \in \mathbb{R}$ be such that $t \in \tilde{\mathbb{T}}^{\sigma\left(t_{0}\right)}$. Then

$$
\left({\stackrel{R}{R} L_{0}}_{t_{0}}^{-\alpha} \nabla_{h}^{-\alpha} \hat{h}_{\beta}\left(t, t_{0}\right)=\hat{h}_{\alpha+\beta}\left(t, t_{0}\right) .\right.
$$

Lemma 2.7. [31]. Let $0<\alpha \leq 1, \beta \in \mathbb{R}$ and $t \in \tilde{\mathbb{T}}^{\sigma^{2}\left(t_{0}\right)}$. Then

$$
\left(\begin{array}{lr}
C \\
t_{0}
\end{array} \nabla_{h}^{\alpha}\right) \hat{h}_{\beta}\left(t, t_{0}\right)=\left\{\begin{array}{lr}
\hat{h}_{\beta-\alpha}\left(t, t_{0}\right), & \beta \neq 0, \\
0, & \beta=0 .
\end{array}\right.
$$

For applications adequate $h$-Mittag-Leffler function is often necessary.

Definition 2.9. Let $\alpha, \beta, \lambda \in \mathbb{R}$. The h-Mittag-Leffler function $E_{\alpha, \beta}^{s, \lambda}(t)$ is defined by

$$
E_{D, \alpha, \beta}^{s, \lambda}(t):=\sum_{k=0}^{\infty} \lambda^{k} \hat{h}_{\alpha k+\beta-1}(t, s)\left(=\sum_{k=0}^{\infty} \lambda^{k} \frac{(t-s)_{h}^{(\alpha k+\beta-1)}}{\Gamma(\alpha k+\beta)}\right),
$$

for $t, s \in \tilde{\mathbb{T}}^{\sigma\left(t_{0}\right)}$ and $t \geq s$. 
Theorem 2.8. Let $\eta \in \mathbb{R}^{+}$and $t \in \tilde{\mathbb{T}}^{\sigma\left(t_{0}\right)}$. Then

$$
\left({ }_{t_{0}}^{R L} \nabla_{h}^{-\eta}\right) E_{D, \alpha, \beta}^{t_{0}, \lambda}(t)=E_{D, \alpha, \beta+\eta}^{t_{0}, \lambda}(t) .
$$

Theorem 2.9. Let $0<\gamma \leq 1$ and let $\alpha k+\beta-1 \neq 1$, for all $k \in \mathbb{N}$. If $t \in \tilde{\mathbb{T}}^{\sigma^{2}\left(t_{0}\right)}$, then

$$
\left(\begin{array}{l}
C \\
t_{0}
\end{array} \nabla_{h}^{\gamma}\right) E_{D, \alpha, \beta}^{t_{0}, \lambda}(t)=\left\{\begin{array}{l}
E_{D, \alpha, \beta-\gamma}^{t_{0}, \lambda}(t), \\
\lambda E_{D, \alpha, \alpha+\beta-\gamma}^{t_{0}, \lambda}(t),
\end{array}\right.
$$

By Definition 2.7 and results presented in (Chapters 1 and 4) in reference [40] (Chapters 1 and 4), if fixed values of $t_{0}$ and $t$ are used, while reducing the value of $h$ so that natural number $\frac{t-t_{0}}{h}$ in this process tends to infinity (or, equivalent, $h \rightarrow 0$ ) for a given discrete function $f_{D}(t)$, if the previous procedure is possible, then $\left({ }_{t_{0}}^{R L} \nabla_{h}^{-\alpha}\right) f_{D}(t) \rightarrow\left(\begin{array}{l}R L \\ t_{0}\end{array} I_{t}^{\alpha}\right) f_{C}(t)$ (function $f_{C}(t)$ is a continual version of $\left.f_{D}(t)\right)$. Also, when $h \rightarrow 0$, then $\left({ }_{t_{0}}^{C} \nabla_{h}^{\alpha}\right) f_{D}(t) \rightarrow$ $\left({ }_{t_{0}}^{C} D_{t}^{\alpha}\right) f_{C}(t)$ and $\mathbb{T}$ is transformed in $\mathbb{R}$. This standard procedure is named "continualization".

\section{The basics of the MSGDETM}

The fractional discrete TSC characteristics presented in the previous section, indicate several shortcomings. The first one is the absence of multiplication of $h$-factorial function, as for polynomial functions (condition $v$ in the Lemma 2.1). Second is the lack of the possibility of formulating generalized discrete Taylors formula (due to the conditions after Definition 2.5; especially, if $0 \varangle \alpha \leq 1$ and $h>0$, then $0_{h}^{(k \alpha)} \neq 0$ and $0_{h}^{(0)}=1$ ). Due to the above mentioned mathematical model weaknesses, MSGDETM could not be formulated. The aim of this section is to formulate the correct product of $h$-factorial function and describe necessary extensions of the operator $h$-fractional Caputo derivative and corresponding nabla $h$-fractional Riemann-Liouville integral. This means that, the generalized discrete Taylor series must be well defined (the elements of the series tend to zero, if variable tends to a given value), and for $h \rightarrow 0$ MSGDETM transforms into MSGDTM (continualization).

In this respect, the adapted and normalized extension based on the dis- 
crete monomials $\hat{h}_{n}(t, s), t, s \in \mathbb{T}, t \geq s$, is discussed in the form

$$
\hat{h}_{\alpha}^{\prime}(t, s):=\hat{h}_{\alpha}(t, s)-\hat{h}_{\alpha}(s, s), \quad \alpha \in \mathbb{R},
$$

or, as $\hat{h}_{\alpha}^{\prime}(t, s):=\frac{\left((t-s)_{h}^{(\alpha)}-0_{h}^{(\alpha)}\right)}{\Gamma(\alpha+1)}$. Further, the features of discrete generalized Taylor series expansion for the function $f_{D}: \mathbb{T} \rightarrow \mathbb{R}, \mathbb{T}=h \mathbb{Z}, h>0$, $t \in\left[t_{0}, t_{r}\right] \subset \mathbb{T}$ are discussed in the form

$$
f_{D}(t)=f_{D}\left(t_{0}\right)+\sum_{k=1}^{K_{D}} F_{D, \alpha}(k) \hat{h}_{k \alpha}^{\prime}\left(t, t_{0}\right), \quad 0<\alpha \leq 1
$$

It is possible that $K_{D} \rightarrow \infty$. By the assumption, the function $f_{D}(t)$, together with extensions of $h$-fractional Caputo derivative, have no singularities i.e., they are $r d$ - and $l d$-continuous and the equation (17) may be written in the form

$$
f_{D}(t)=f_{D}\left(t_{0}\right)+\sum_{k=1}^{K_{D}} F_{D, \alpha}^{\prime}(k)\left(\left(t-t_{0}\right)_{h}^{(k \alpha)}-0_{h}^{(k \alpha)}\right),
$$

where $F_{D, \alpha}^{\prime}(k)=\frac{F_{D, \alpha}(k)}{\Gamma(k \alpha+1)}$.

The following definition describes the properties of the new operation of the multiplication between non-normalized and adapted extensions of monomials based on its discrete versions.

Definition 3.1. Let $t_{0}, t \in \mathbb{T}, t>t_{0}$ and $h>0$.

(i) The set of adapted monomials $S_{D}$ and binary operation, h-product of adapted monomials: $\otimes: S_{D} \times S_{D} \rightarrow S_{D}$. are defined in the following way:

$$
\left(\left(t-t_{0}\right)_{h}^{(\alpha)}-0_{h}^{(\alpha)}\right) \otimes\left(\left(t-t_{0}\right)_{h}^{(\beta)}-0_{h}^{(\beta)}\right):=\left(\left(t-t_{0}\right)_{h}^{(\alpha+\beta)}-0_{h}^{(\alpha+\beta)}\right) .
$$

$$
S_{D}:=\left\{\left(\left(t-t_{0}\right)_{h}^{(\gamma)}-0_{h}^{(\gamma)}\right), \gamma \in \mathbb{R}^{+}\right\}
$$

(ii) Product $\left(\left(t-t_{0}\right)_{h}^{(\alpha)}-0_{h}^{(\alpha)}\right)$ with real constant is defined in the standard way.

(iii) If $h \rightarrow 0$, then $S_{D} \rightarrow S_{C}=\left\{\left(t-t_{0}\right)^{\gamma}, \gamma \in \mathbb{R}\right\}$. In this case, $\otimes$ becomes standard multiplication of monomials. 
$S_{D}$ and operation $\otimes,\left(S_{D}, \otimes\right)$ is a commutative semigroup. The addition and subtraction of adapted monomials are defined in a standard way.

Definition 3.2. Let $\alpha \in(0,1], h>0, t_{0} \in \mathbb{T}, t \in \tilde{\mathbb{T}}^{\sigma\left(t_{0}\right)}$. The left adapted $\alpha$-th, $h$-fractional derivative an Caputo sense of function $\hat{h}_{\beta}^{\prime}\left(t, t_{0}\right), \beta \in \mathbb{R}$, defined by

$$
\begin{array}{rc}
\left(\begin{array}{l}
C \\
t_{0}
\end{array} \boldsymbol{\nabla}_{h}^{\alpha}\right) \hat{h}_{\beta}^{\prime}\left(t, t_{0}\right):=0, & \beta=0, \\
\left(\begin{array}{l}
C \\
t_{0}
\end{array} \mathbf{\nabla}_{h}^{\alpha}\right) \hat{h}_{\beta}^{\prime}\left(t, t_{0}\right):=\hat{h}_{\beta-\alpha}^{\prime}\left(t, t_{0}\right), & \beta \neq 0 .
\end{array}
$$

The left adapted 0 -th $h$-fractional derivative of function $f_{D}(t) \in \mathcal{F}_{\mathbb{T}}$ satisfying equation (17) is $\left(\begin{array}{l}C \\ t_{0}\end{array} \mathbf{\nabla}_{h}^{0}\right) f_{D}(t):=f_{D}(t)$. The $m \alpha$-th derivative of function $f_{D}(t)$, for $t \in \tilde{\mathbb{T}}^{\sigma^{m+1}\left(t_{0}\right)}$, is

$$
\left(\begin{array}{l}
C \\
t_{0}
\end{array} \mathbf{\nabla}_{h}^{m \alpha}\right) f_{D}(t):=\left(\begin{array}{l}
C \\
t_{0}
\end{array} \mathbf{\nabla}_{h}^{\alpha}\right)\left(\begin{array}{l}
C \\
t_{0}
\end{array} \mathbf{\nabla}_{h}^{(m-1) \alpha}\right) f_{D}(t), \quad m \in \mathbb{N} .
$$

In a similar way is defined, for $m \in \mathbb{N}_{0},\left(\begin{array}{l}C \\ t_{0}\end{array} \nabla_{h}^{m \alpha}\right) \hat{h}_{\beta}^{\prime}\left(t, t_{0}\right)$.

Analogously to Definition 2.9, following definition could be introduced:

Definition 3.3. Let $\alpha, \beta, \lambda \in \mathbb{R}$. The $h-$ Mittag-Leffler discrete adapted function $E_{D, \alpha, \beta}^{a, s, \lambda}(t)$ is defined by

$$
E_{D, \alpha, \beta}^{a, s, \lambda}(t):=\sum_{k=0}^{\infty} \lambda^{k} \hat{h}_{\alpha k+\beta-1}^{\prime}(t, s),
$$

for $t, s \in \tilde{\mathbb{T}}^{\sigma\left(t_{0}\right)}$ and $t \geqslant s$.

Theorem 3.1. Properties of some functions $f_{D}(t)$ or $\hat{h}_{\beta}^{\prime}\left(t, t_{0}\right)$, when acting on them $\left({ }_{t 0}^{C} \nabla_{h}^{m \alpha}\right), m \in \mathbb{N}$, with $K_{D} \rightarrow \infty$.

Let $\alpha \in(0,1], h>0, t_{0} \in \mathbb{T}$.

(i) If $\beta \neq 0$ and $t \in \tilde{\mathbb{T}}^{\sigma\left(t_{0}\right)}$, then $\left(\begin{array}{l}C \\ t_{0}\end{array} \boldsymbol{\nabla}_{h}^{\alpha}\right) \hat{h}_{\beta}^{\prime}\left(t, t_{0}\right)=\left(\begin{array}{l}C \\ t_{0}\end{array} \nabla_{h}^{\alpha}\right) \hat{h}_{\beta}^{\prime}\left(t, t_{0}\right)-$ $\hat{h}_{\beta-\alpha}^{\prime}\left(t_{0}, t_{0}\right)$.

(ii) In relation to (17), for $t \in \tilde{\mathbb{T}}^{\sigma^{m+1}\left(t_{0}\right)}$, follow $\left(t_{0}^{C} \mathbf{\nabla}_{h}^{m \alpha}\right)\left(f_{D}\left(t_{0}\right)\right)=\delta_{k m} F_{D, \alpha}(k)$, where $\delta_{k m}$ is a Kronecker delta.

(iii) For $t \in \tilde{\mathbb{T}}^{\sigma\left(t_{0}\right)},\left(\begin{array}{l}C \\ t_{0}\end{array} \mathbf{\nabla}_{h}^{\alpha}\right) E_{\alpha, 1}^{a, t_{0}, \lambda}(t)=\lambda E_{\alpha, 1}^{a, t_{0}, \lambda}(t)$.

Proof. All the proofs used Lemma 2.7, (16), (17) and Definitions 3.1, 3.2, 3.3. 
Important relation between the operators $\left(\begin{array}{l}C \\ t_{0}\end{array} \nabla_{h}^{\alpha}\right)$ and $\left(\begin{array}{l}C \\ t_{0}\end{array} \boldsymbol{\nabla}_{h}^{\alpha}\right)$, makes the following statement.

Theorem 3.2. If $\alpha \in(0,1]$ over the time scale interval $\left[t_{0}, t\right] \cap \tilde{\mathbb{T}}^{t_{0}}$ for the series (17), when $K_{D} \rightarrow \infty$. Then

$$
\left(\begin{array}{l}
C \\
t_{0}
\end{array} \boldsymbol{\nabla}_{h}^{\alpha}\right) f_{D}(t)=\left({ }_{t_{0}}^{C} \nabla_{h}^{\alpha}\right) f_{D}(t)-\sum_{k=2}^{\infty} F_{D, \alpha}(k) \hat{h}_{(k-1) \alpha}\left(t_{0}, t_{0}\right) .
$$

Proof. Proof follows from relations

$$
\left(\begin{array}{l}
C \\
t_{0}
\end{array} \boldsymbol{\nabla}_{h}^{\alpha}\right) f_{D}(t)=F_{D, \alpha}(1)+\sum_{k=2}^{\infty} F_{D, \alpha}(k) \hat{h}_{(k-1) \alpha}^{\prime}\left(t, t_{0}\right),
$$

by the Definition 3.2 and, for Lemma 2.7,

$$
\left(\begin{array}{l}
C \\
t_{0}
\end{array} \nabla_{h}^{\alpha}\right) f_{D}(t)=F_{D, \alpha}(1)+\sum_{k=2}^{\infty} F_{D, \alpha}(k) \hat{h}_{(k-1) \alpha}\left(t, t_{0}\right) .
$$

Due to the introduced operator $\left(\begin{array}{c}C \\ t_{0}\end{array} \nabla_{h}^{\alpha}\right)$, we must define a new nabla $h$ fractional Riemann-Liouville integral of order $\alpha \in(0,1]:\left(\begin{array}{l}R L \\ t_{0}\end{array} \mathbf{\nabla}_{h}^{-\alpha}\right)$.

Definition 3.4. The left adapted nabla h-fractional integral (in the sense of Riemann-Liouville) of order $\alpha \in(0,1]$ over the time scale interval $\tilde{\mathbb{T}}^{t_{0}}$, for the functions $\hat{h}_{\beta}^{\prime}\left(t, t_{0}\right), \beta \in \mathbb{R}$, and for those $f_{D}(t)$ that satisfy (17), when $K_{D} \rightarrow \infty$, is defined by

$$
\begin{gathered}
\left(_{t_{0}}^{R L} \mathbf{\nabla}_{h}^{-\alpha}\right) \hat{h}_{\beta}^{\prime}\left(t, t_{0}\right):=\hat{h}_{\alpha+\beta}^{\prime}\left(t, t_{0}\right), \\
\left(\begin{array}{c}
R L \mathbf{\nabla}_{h}^{-\alpha} \\
t_{0}
\end{array}\right) f_{D}(t):=f_{D}\left(t_{0}\right) \hat{h}_{\alpha}^{\prime}\left(t, t_{0}\right)+\sum_{k=1}^{\infty} F_{D, \alpha}(k) \hat{h}_{(k+1) \alpha}^{\prime}\left(t, t_{0}\right), \\
\left(\begin{array}{l}
R L \\
t_{0}
\end{array} \nabla_{h}^{+m \alpha}\right) f_{D}(t):=\left(\left(\begin{array}{l}
R L \\
t_{0}
\end{array} \mathbf{\nabla}_{h}^{-\alpha}\right)\left(\begin{array}{l}
R L \\
t_{0}
\end{array} \mathbf{\nabla}_{h}^{-(m-1) \alpha}\right)\right) f_{D}(t),
\end{gathered}
$$

where $m \in \mathbb{N} \backslash\{1\}$. For $m=0$ is $\left(\begin{array}{c}R L \\ t_{0}\end{array} \mathbf{\nabla}_{h}^{0}\right) f_{D}(t):=f_{D}(t)$. Also, in a similar way is defined, for $m \in \mathbb{N}_{0},\left(\begin{array}{l}R L \\ t_{0}\end{array} \mathbf{\nabla}_{h}^{-m \alpha}\right) \hat{h}_{\beta}^{\prime}\left(t, t_{0}\right)$.

Theorem 3.3. If $\alpha \in(0,1]$, for functions satisfying (17), when $K_{D} \rightarrow \infty$, following relations are valid

$$
\left(\left(\begin{array}{l}
C \\
t_{0}
\end{array} \boldsymbol{\nabla}_{h}^{\alpha}\right)\left(\begin{array}{l}
R L \\
t_{0}
\end{array} \mathbf{\nabla}_{h}^{-\alpha}\right)\right) f_{D}(t)=f_{D}(t)
$$


and

$$
\left(\left(\begin{array}{l}
R L \\
t_{0}
\end{array} \mathbf{\nabla}_{h}^{-\alpha}\right)\left(\begin{array}{l}
C \\
t_{0}
\end{array} \mathbf{\nabla}_{h}^{\alpha}\right)\right) f_{D}(t)=f_{D}(t)-f_{D}\left(t_{0}\right) .
$$

Proof. For Definition 3.4,

$$
\begin{aligned}
& \left(\left(\begin{array}{c}
C \\
t_{0}
\end{array} \mathbf{\nabla}_{h}^{\alpha}\right)\left(\begin{array}{l}
R L \\
t_{0}
\end{array} \mathbf{\nabla}_{h}^{-\alpha}\right)\right) f_{D}(t)= \\
& \quad\left(\begin{array}{l}
C \\
t_{0}
\end{array} \mathbf{\nabla}_{h}^{\alpha}\right)\left(f_{D}\left(t_{0}\right) \hat{h}_{\alpha}^{\prime}\left(t, t_{0}\right)+\sum_{k=1}^{\infty} F_{D, \alpha}(k) \hat{h}_{(k+1) \alpha}^{\prime}\left(t, t_{0}\right)\right)
\end{aligned}
$$

Result (19) is then obtained by using Definition 3.2 and Theorem 2.9. Reverse order of proof is for Eq. (20). exists.

Also important relation between the operators $\left(\begin{array}{l}R L \\ t_{0}\end{array} \nabla_{h}^{-\alpha}\right)$ and $\left(\begin{array}{l}R L \\ t_{0}\end{array} \mathbf{\nabla}_{h}^{-\alpha}\right)$

Theorem 3.4. If $\alpha \in(0,1]$, for the functions satisfy (17), when $K_{D} \rightarrow \infty$, following relation is valid

$$
\begin{aligned}
& \quad\left(\begin{array}{l}
R L \\
t_{0}
\end{array} \nabla_{h}^{-\alpha}\right) f_{D}(t)=\left(\begin{array}{l}
R L \\
t_{0}
\end{array} \nabla_{h}^{-\alpha}\right) f_{D}(t)+f_{D}\left(t_{0}\right) \hat{h}_{\alpha}\left(t_{0}, t_{0}\right)+ \\
& \left.\sum_{k=1}^{\infty} F_{D, \alpha}(k) \hat{h}_{(k+1) \alpha}\left(t_{0}, t_{0}\right)\right)-y \\
& \left.\hat{h}_{\alpha}\left(t, t_{0}\right) \sum_{k=1}^{\infty} F_{D, \alpha}(k) \hat{h}_{k \alpha}\left(t_{0}, t_{0}\right)\right) .
\end{aligned}
$$

Proof. Proof follows from the relation Eq. (16)

$$
\begin{aligned}
& \left(\begin{array}{c}
R L \\
t_{0}
\end{array} \nabla_{h}^{-\alpha}\right) f_{D}(t)=f_{D}\left(t_{0}\right) \hat{h}_{\alpha}\left(t, t_{0}\right)+ \\
& \sum_{k=1}^{\infty} F_{D, \alpha}(k)\left[\hat{h}_{(k+1) \alpha}\left(t, t_{0}\right)-\hat{h}_{\alpha}\left(t, t_{0}\right) \hat{h}_{k \alpha}\left(t_{0}, t_{0}\right)\right]
\end{aligned}
$$

by/the Lemma 2.6 and Definition 3.4.

Corollary 3.5. The adapted and normalized generalized monomials $\hat{h}_{n \alpha}^{\prime}$ : $\mathbb{T} \times \mathbb{T} \rightarrow \mathbb{R}, n \in \mathbb{N}_{0}, \alpha \in(0,1]$, are given by

$$
\hat{h}_{n \alpha}^{\prime}(t, s)=\left\{\begin{array}{lr}
1, & n=0 \\
\left(\begin{array}{l}
R L \\
s
\end{array} \mathbf{\nabla}_{h}^{-\alpha}\right) \hat{h}_{(n-1) \alpha}^{\prime}(t, s), & n \in \mathbb{N}
\end{array}\right.
$$


for $s, t \in \mathbb{T}$ and $t \geq s$. Previous statement means that $\left(\underset{t_{0}}{C} \boldsymbol{\nabla}_{h}^{\alpha}\right) \hat{h}_{n \alpha}^{\prime}(t, s)=$ $\hat{h}_{(n-1) \alpha}^{\prime}(t, s)$.

Theorem 3.6. Discrete generalized Taylor's formula. Let $n \in \mathbb{N}$ and $t, t_{0} \in$ $\mathbb{T}$. Suppose $f_{D}: \mathbb{T} \rightarrow \mathbb{R}, t \in \tilde{\mathbb{T}}^{\sigma^{n+1}\left(t_{0}\right)}$, it is possible $K_{D} \rightarrow \infty$, then

$$
\begin{gathered}
f_{D}(t)=f_{D}\left(t_{0}\right)+\sum_{k=1}^{n} \hat{h}_{k \alpha}^{\prime}\left(t, t_{0}\right)\left(\begin{array}{l}
C \\
t_{0}
\end{array} \boldsymbol{\nabla}_{h}^{k \alpha}\right) f_{D}\left(t_{0}\right)+ \\
R_{D, \alpha}(n+1, t),
\end{gathered}
$$

where $R_{D, \alpha}(n+1, t)=\left({ }_{t_{0}}^{R L} \mathbf{\nabla}_{h}^{-(n+1) \alpha}\right)\left({ }_{t_{0}}^{C} \mathbf{\nabla}_{h}^{(n+1) \alpha}\right) f_{D}(t)$.

Proof. In addition, for $m \in \mathbb{N}$, valid

$$
\begin{aligned}
& \left(\begin{array}{l}
R L \\
t_{0}
\end{array} \mathbf{\nabla}_{h}^{-m \alpha}\right)\left(\begin{array}{l}
C \\
t_{0}
\end{array} \mathbf{\nabla}_{h}^{m \alpha}\right) f_{D}(t)= \\
& f_{D}(t)-\left(f_{D}\left(t_{0}\right)+\sum_{k=1}^{m} \hat{h}_{k \alpha}^{\prime}\left(t, t_{0}\right)\left({ }_{t_{0}}^{C} \mathbf{\nabla}_{h}^{k \alpha}\right) f_{D}\left(t_{0}\right)\right) .
\end{aligned}
$$

Also the next relation is valid

$$
R_{D, \alpha}(n, t)-R_{D, \alpha}(n+1, t)=\hat{h}_{n \alpha}^{\prime}\left(t, t_{0}\right)\left(\begin{array}{l}
C \\
t_{0}
\end{array} \mathbf{\nabla}_{h}^{n \alpha}\right) f_{D}\left(t_{0}\right) .
$$

From the relation (22), come next:

$$
\sum_{k=0}^{n} R_{D, \alpha}(k, t)-R_{D, \alpha}(k+1, t)=f_{D}\left(t_{0}\right)+\sum_{i=1}^{n} \hat{h}_{i \alpha}^{\prime}\left(t, t_{0}\right)\left({ }_{t_{0}}^{C} \mathbf{\nabla}_{h}^{i \alpha}\right) f_{D}\left(t_{0}\right)
$$

that is

$$
f_{D}(t)-R_{D, \alpha}(n+1, t)=f_{D}\left(t_{0}\right)+\sum_{i=1}^{n} \hat{h}_{i \alpha}^{\prime}\left(t, t_{0}\right)\left(\begin{array}{l}
C \\
t_{0}
\end{array} \mathbf{\nabla}_{h}^{i \alpha}\right) f_{D}\left(t_{0}\right) .
$$

In the following text, Cauchy type fractional difference initial value problem will be considered, analogous to (9)

$$
\left(\begin{array}{l}
C \\
0
\end{array} \mathbf{\nabla}_{h}^{\alpha}\right) f_{D}(t)=G_{D}\left(t, f_{D}\right)
$$


$G_{D}\left(t, f_{D}\right)$ is a real function, together with the initial condition $f_{D}(0)=$ $c_{D, \alpha, 0}$. In general, it is considered analogous to [31]. For example, in accordance to Theorem 3.1, $\left.{ }_{0}^{C} \nabla_{h}^{\alpha}\right) f_{D}(t)=\lambda f_{D}(t)$ has the next solution: $f_{D}(t)=c_{D, \alpha, 0} E_{D, \alpha, 1}^{a, 0, \lambda}(t)$. It is assumed that it may be resolved and systems of nonlinear equations are based on $\left(\begin{array}{c}C \\ 0\end{array} \boldsymbol{\nabla}_{h}^{\alpha}\right)$.

If $\alpha=1$ in (21), Theorem 2.2 could be obtained. Theorem 3.6 is the basic result of this research. It represents the foundation of the MSGDETM. Accordingly, it is possible to conduct the continualization procedure. Therefore, if $h \rightarrow 0$, taking into account Theorems 3.2 and 3.4, then $\left(\begin{array}{c}R L \\ t_{0}\end{array} \mathbf{\nabla}_{h}^{-\alpha}\right) \rightarrow\left(\begin{array}{c}R L \\ t_{0}\end{array} I_{t}^{\alpha}\right),\left(\begin{array}{c}C \\ t_{0}\end{array} \mathbf{\nabla}_{h}^{\alpha}\right) \rightarrow\left(\begin{array}{c}C \\ t_{0}\end{array} D_{t}^{\alpha}\right)$ and $f_{D}(t) \rightarrow f_{C}(t)$. Also, (22) tends to $R_{C, \alpha}(n+1, t)-R_{C, \alpha}(n, t)=\frac{\left(t-t_{0}\right)^{n \alpha}}{\Gamma(n \alpha+1)}\left({ }_{t_{0}}^{C} D_{t}^{n \alpha}\right) f_{C}\left(t_{0}\right), R_{C, \alpha}(n, t)=$

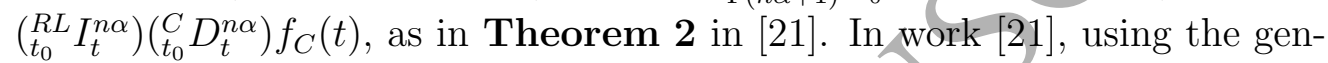
eralized mean value theorem (Theorem $\mathbf{1}$ ), the generalized Taylors formula (Theorem 3) is proved. Therefore, MSGDETM transforms into MSGDTM.

The multistep generalized difference transformation method is treated as an algorithm in a sequence of small discrete sets of points in time for finding accurate approximate solutions over a longer time frame, similarly as in the reference [14]. In actual applications of the MSGDETM, the function $f_{D}(t)$ is expressed by a finite series, and, for $t_{0}=0,(18)$ can be presented as

$$
f_{D}(t) \approx F_{D, \alpha}^{\prime}(0)+\sum_{n=1}^{N} F_{D, \alpha}^{\prime}(n)\left(t_{h}^{(n \alpha)}-0_{h}^{(n \alpha)}\right) .
$$

Here, $N$ is decided by the convergence of accepted regularity.

Table 1

Operations of difference transformation.

\begin{tabular}{ll}
\hline Original function & Transformed function \\
\hline$f_{D}(t)=f_{D 1}(t) \pm f_{D 2}(t)$ & $F_{D, \alpha}^{\prime}(n)=F_{D, \alpha, 1}^{\prime}(n) \pm F_{D, \alpha, 2}^{\prime}(n)$ \\
$f_{D}(t)=c \cdot f_{D 1}(t)$ & $F_{D, \alpha}^{\prime}(n)=c \cdot F_{D, \alpha, 1}^{\prime}(n)$ \\
$f_{D}(t)=f_{D 1}(t) \otimes f_{D 2}(t)$ & $F_{D, \alpha}^{\prime}(n)=\sum_{l=0}^{n} F_{D, \alpha, 1}^{\prime}(l) \cdot F_{D, \alpha, 2}^{\prime}(n-l)$ \\
$f(t)=\left(\begin{array}{l}C \\
0\end{array} \mathbf{\nabla}_{h}^{\alpha}\right) f_{D 1}(t)$ & $F_{D, \alpha}^{\prime}(n)=\frac{\Gamma(n+1) \alpha+1)}{\Gamma(n \alpha+1)} F_{D, \alpha, 1}^{\prime}(n+1)$ \\
$f_{D}(t)=\left(\begin{array}{l}R L \\
0\end{array} \mathbf{\nabla}_{h}^{-\alpha}\right) f_{D 1}(t)$ & $F_{D, \alpha}^{\prime}(n)=\frac{\Gamma((n-1) \alpha+1)}{\Gamma(n \alpha+1)} F_{D, \alpha, 1}^{\prime}(n-1)$ \\
$f_{D}(t)=t_{h}^{(m \alpha)}-0_{h}^{(m \alpha)}$ & $F_{D, \alpha}^{\prime}(n)=\delta_{n m}$ \\
\hline
\end{tabular}

The fundamental operations performed by the generalized difference transformation can easily be obtained and are listed in Table 1 . In order to charac- 
terize the Cauchy type fractional difference initial value problem (23) through the MSGDETM, it is necessary to introduce some concepts. First, discrete analog of the set $[0, T]$, with the above partition $\mathcal{P}$, for $M \in \mathbb{N}, \Delta t=\frac{T}{M}$, $t_{m}=t_{m-1}+\Delta t, t_{0}=0$ and $t_{m n}=t_{m-1}+n \frac{\Delta t}{N}, N \in \mathbb{N}, t_{00}=t_{0}, t_{m 0}=t_{m-1}$, $h=t_{m n}-t_{m n-1}$ (in total there are $N M+1$ equidistant points: $t_{00}, t_{n m}=$ $\left.t_{00}+n m h\right)$ is introduced in the following way: $\mathcal{P}^{\prime}$ is a set of elements containing the points defined by the following sets: $I_{1}=\left\{0, h, \ldots, t_{1}-h\right\}, I_{2}=$ $\left\{t_{1}, t_{1}+h, \ldots, t_{2}-h\right\}, \ldots, I_{M}=\left\{t_{M-1}, t_{M-1}+h, \ldots, t_{M}-h\right\}$, and point $t_{M}$. In addition, for the basic step $h$, general step $h^{\prime}=\nu h, \nu \in\{1,2, \ldots, N\}$, such that $N^{\prime}=\frac{N}{\nu} \in \mathbb{N}$ is defined. It can be noticed that, by definition, $h \leq h^{\prime} \leq N h$. Now, if the $\mathbb{T}=\mathcal{P}^{\prime}$ and $h^{\prime}=h$, MSGDETM gives the exact solution. If $\mathcal{P}^{\prime} \subset \mathbb{T}=h \mathbb{Z}$, then it is always a numerical solution. The general step $h^{\prime}$, for the MSGDETM and MSGDTM, determines the quality of the numerical approximation.

\section{MSGDETM numerical experiments for the fractional discrete Michaelis-Menten model in Caputo sense}

After the introduction of a new discrete fractional calculus on time scales and the new numerical method, it is necessary an appropriate model to be defined. A mechanism of the Michaelis-Menten kinetic reaction for this calculus has the following form

$$
\begin{array}{r}
\left(\begin{array}{l}
C \\
0
\end{array} \mathbf{\nabla}_{h}^{\alpha 1}\right) u_{D}=-\varepsilon u_{D}+\varepsilon u_{D} \otimes v_{D}+\varepsilon\left(k_{3}-\lambda\right) v_{D} \\
\left(\begin{array}{l}
C \\
0
\end{array} \mathbf{\nabla}_{h}^{\alpha_{2}}\right) v_{D}=u_{D}-u_{D} \otimes v_{D}-k_{3} v_{D} \\
\left(\begin{array}{l}
C \\
0
\end{array}\right. \\
\left.\mathbf{\nabla}_{h}^{\alpha_{3}}\right) w_{D}=\lambda v_{D} \\
u_{D}(0)=1, v_{D}(0)=0, w_{D}(0)=0 .
\end{array}
$$

Relations (24) are a generalization of Equations (8), (9), (10) and (11) presented in reference [14]. This paper considers the case when $0<\alpha_{i} \leq 1$ for $i=1,2,3$. The model described in (24) assumed discrete fractional time differences with respect to the concentrations $u_{D}, v_{D}$ and $w_{D}$. If these time differences are realized as the generalized velocities, then they are extensions of classical concepts of continuous velocities. Properties of these continuous velocities are described in terms of Boltzmann-Gibbs statistical thermodynamics. We could make the assumption that the generalized velocities are described by some wider concept. For this approach, if it is possible at all, the same idea as for Tsallis statistics [41] should apply: the new theory is 
not intended to replace Boltzmann-Gibbs statistics, but rather supplement it instead. In the general case, for the TSC, a time scale is defined to be an arbitrary closed subset of the real numbers $\mathbb{R}$, with the standard inherited topology, and it is the final level, which is considered. Regarding to that, the assumption of reaction (ir)reversibility in the case when the product is discretely removed, in fractional difference Michaelis-Menten reaction as in Boltzmann-Gibbs statistical thermodynamics, is a still open.

MSGDETM is applied in order to solve the system of equations (24).

Taking the difference transformation of (24) with respect to time $t$, following equations is obtained:

$$
\begin{aligned}
& \frac{\Gamma\left(\alpha_{1}(n+1)+1\right)}{\Gamma\left(\alpha_{1} n+1\right)} U_{D, \alpha_{1}}^{\prime}(n+1)=-\varepsilon U_{D, \alpha_{1}}^{\prime}(n)+ \\
& \varepsilon\left(\sum_{l=0}^{n} U_{D, \alpha_{1}}^{\prime}(l) V_{D, \alpha_{1}}^{\prime}(n-l)+\left(k_{3}-\lambda\right) V_{D, \alpha_{1}}^{\prime}(n)\right), \\
& V_{D, \alpha_{2}}^{\prime}(n+1)=\frac{\Gamma\left(\alpha_{2} n+1\right)}{\Gamma\left(\alpha_{2}(n+1)+1\right)} \\
& {\left[U_{D, \alpha_{2}}^{\prime}(n)-\sum_{l=0}^{n} U_{D, \alpha_{2}}^{\prime}(l) V_{D, \alpha_{2}}^{\prime}(n-l)-k_{3} V_{D, \alpha_{2}}^{\prime}(n)\right],} \\
& W_{D, \alpha_{3}}^{\prime}(n+1)=\frac{\Gamma\left(\alpha_{3} n+1\right)}{\Gamma\left(\alpha_{3}(n+1)+1\right)} \cdot \lambda V_{D, \alpha_{3}}^{\prime}(n),
\end{aligned}
$$

where $U_{D, \alpha_{i}}^{\prime}(n), V_{D, \alpha_{i}}^{\prime}(n)$ and $W_{D, \alpha_{i}}^{\prime}(n)$, for $i=1,2,3$, are the generalized difference transformation forms of $u_{D}(t), v_{D}(t)$ and $w_{D}(t)$, respectively. For the initial conditions, difference transformation forms are given by $u_{D, \alpha_{i}}(0) \equiv$ $U_{D, \alpha_{i}}^{\prime}(0)=1, v_{D, \alpha_{i}}(0) \equiv V_{D, \alpha_{i}}^{\prime}(0)=0$, and $w_{D, \alpha_{i}}(0) \equiv W_{D, \alpha_{i}}^{\prime}(0)=0$. After the difference transformation series solution for the systems (18) can be 
presented

$$
\begin{gathered}
u_{D, \alpha_{i}}(t)=U_{D, \alpha_{i}}^{\prime}(0)+\sum_{n=1}^{N} U_{D, \alpha_{i}}^{\prime}(n)\left(t_{h}^{\left(n \alpha_{i}\right)}-0_{h}^{\left(n \alpha_{i}\right)}\right), \\
v_{D, \alpha_{i}}(t)=V_{D, \alpha_{i}}^{\prime}(0)+\sum_{n=1}^{N} V_{D, \alpha_{i}}^{\prime}(n)\left(t_{h}^{\left(n \alpha_{i}\right)}-0_{h}^{\left(n \alpha_{i}\right)}\right) \\
w_{D, \alpha_{i}}(t)=W_{D, \alpha_{i}}^{\prime}(0)+\sum_{n=1}^{N} W_{D, \alpha_{i}}^{\prime}(n)\left(t_{h}^{\left(n \alpha_{i}\right)}-0_{h}^{\left(n \alpha_{i}\right)}\right)
\end{gathered}
$$

over the set $\mathcal{P}^{\prime}$. The idea of generalized discrete multistep method expressed by

$$
a_{D, \alpha_{i}}\left((t)=\left\{\begin{array}{l}
a_{D, \alpha_{i}, 1}((t), \\
a_{D, \alpha_{i}, 2}(t), \\
\vdots \\
a_{D, \alpha_{i}, M}((t),
\end{array}\right.\right.
$$

and

$$
a_{D, \alpha_{i}, m}(t)=A_{D, \alpha_{i}, m}^{\prime}(0)+\sum_{n=1}^{N} A_{D, \alpha_{i}, m}^{\prime}(n)\left(\left(t-t_{m-1}\right)_{h}^{\left(n \alpha_{i}\right)}-0_{h}^{\left(n \alpha_{i}\right)}\right) .
$$

For each value of the index $m$, for the functions $U_{D, \alpha_{i}, m}^{\prime}(n), V_{D, \alpha_{i}, m}^{\prime}(n)$ and $W_{D, \alpha_{i}, m}^{\prime}(n)$ are written toidentical relations for $U_{D, \alpha_{i}}^{\prime}(n), V_{D, \alpha_{i}}^{\prime}(n)$ and $W_{D, \alpha_{i}}^{\prime}(n)$ those of (25), respectively. The initial conditions, except $u_{D, \alpha_{i}, 1}(0) \equiv U_{D, \alpha_{i}, 1}^{\prime}(0)=$ $1, v_{D, \alpha_{i}, 1}(0) \equiv V_{D, \alpha_{i}, 1}^{\prime}(0)=0$ and $w_{D, \alpha_{i}, 1}(0) \equiv W_{D, \alpha_{i}, 1}^{\prime}(0)=0$, for $k=$ $2, \ldots, M$ are $a_{D, \alpha_{i}, k}\left(t_{k-1}\right)=a_{D, \alpha_{i}, k-1}\left(t_{k-1}\right)$.

MSGDETM (MSGDTM) provides direct and evident symbolic terms of analytic solutions, as well as numerical approximate solutions to both, linear and nonlinear difference i.e. differential equations. To demonstrate the effectiveness of the proposed algorithm as an approximate tool, the nonlinear system of fractional difference or differential equations (24) as in reference [14] is solved. For this purpose, the proposed algorithms on the interval $[0,10]$, making discretization for different values of $h^{\prime}$ are applied.

In Figures 1 and 2 there are presented numerical solutions for classical Michaelis - Menten kinetic model. Solutions are obtained by using MSDETM 


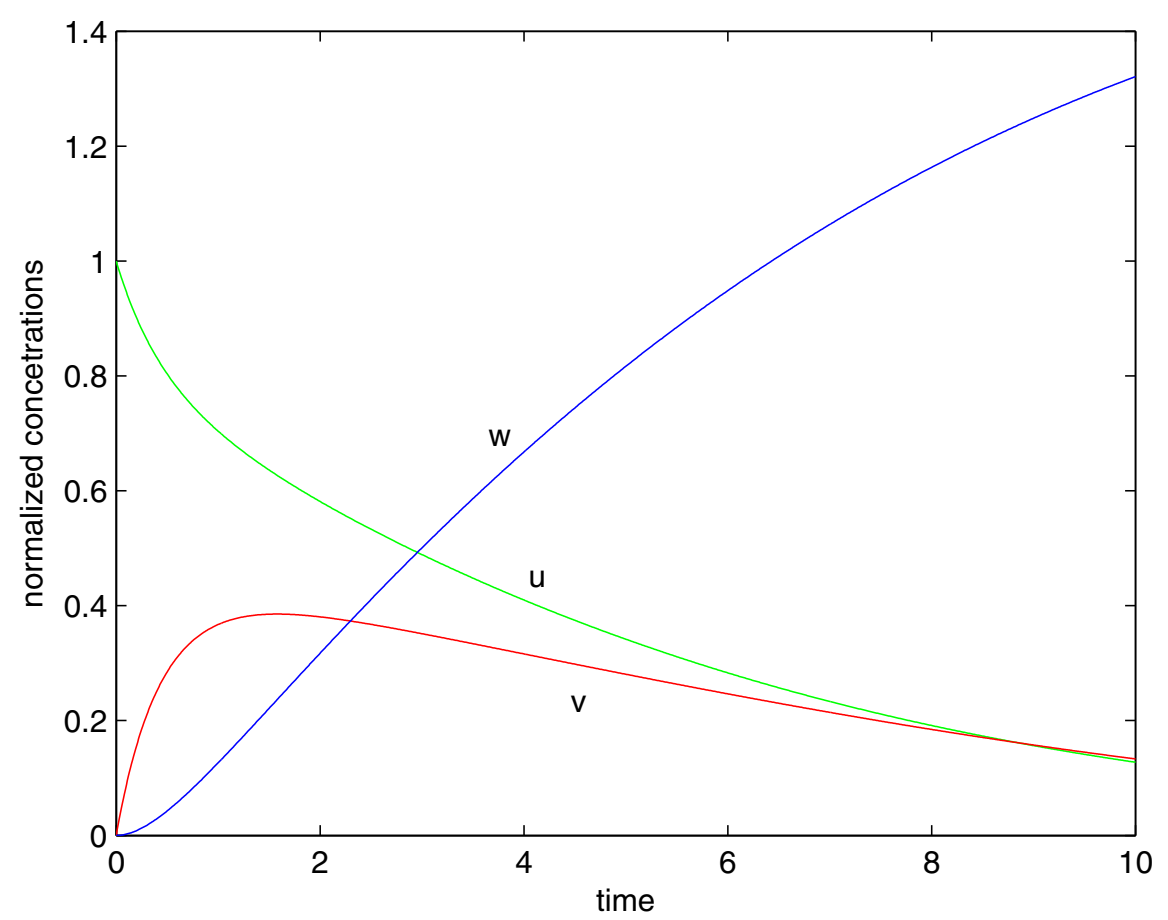

Figure 1: Profile of the normalized concentrations of the substrate $u$, enzyme-substrate complex $v$, and product $w$ for $k_{3}=1, \lambda=0.5, \varepsilon=0.6$, and $\alpha_{i}=1, i=1,2,3$. The same solutions obtained with MSDETM, MSDTM and Runge-Kutta method for model (24). 


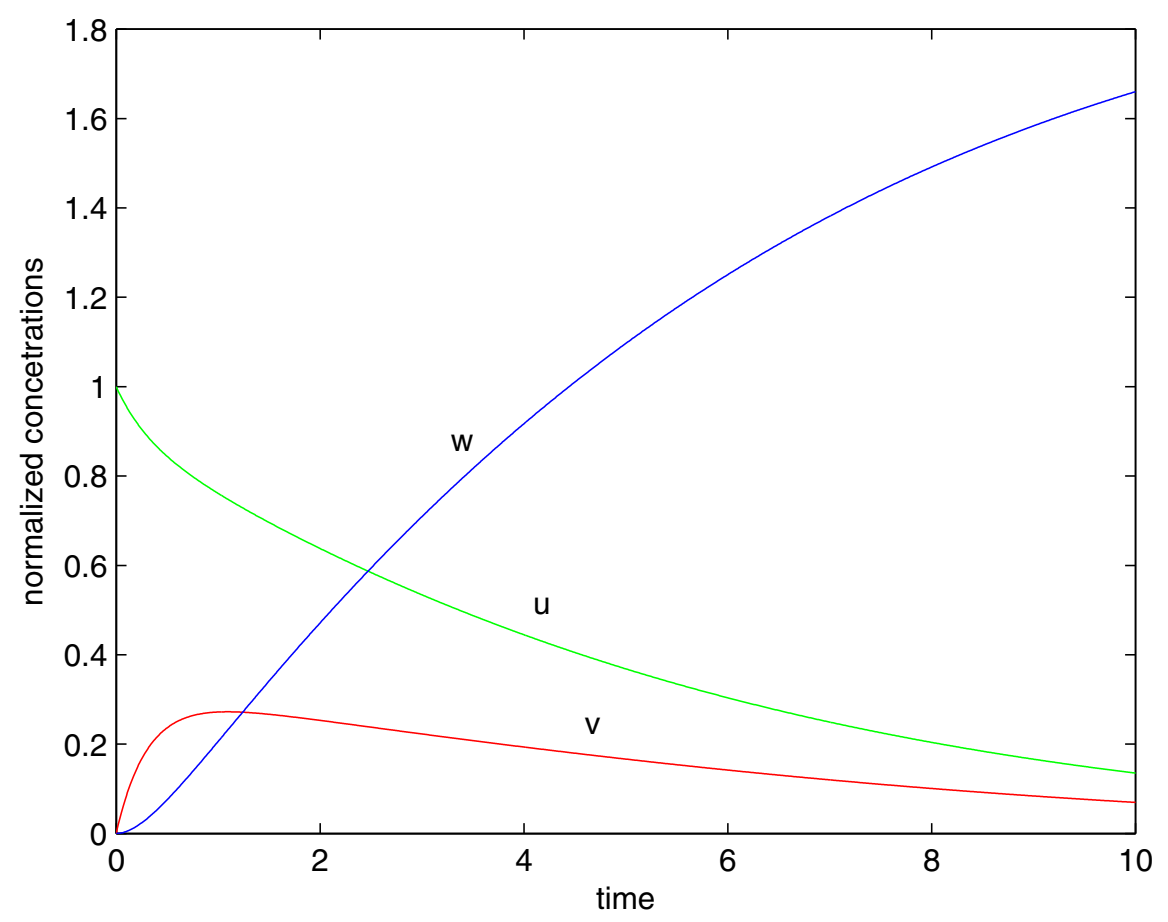

Figure 2: Profile of the normalized concentrations of the substrate $u$, enzyme-substrate complex $v$, and product $w$ for $k_{3}=2, \lambda=1, \varepsilon=0.5$, and $\alpha_{i}=1, i=1,2,3$. The same solutions obtained with MSDETM, MSDTM and Runge-Kutta method for model (24). 


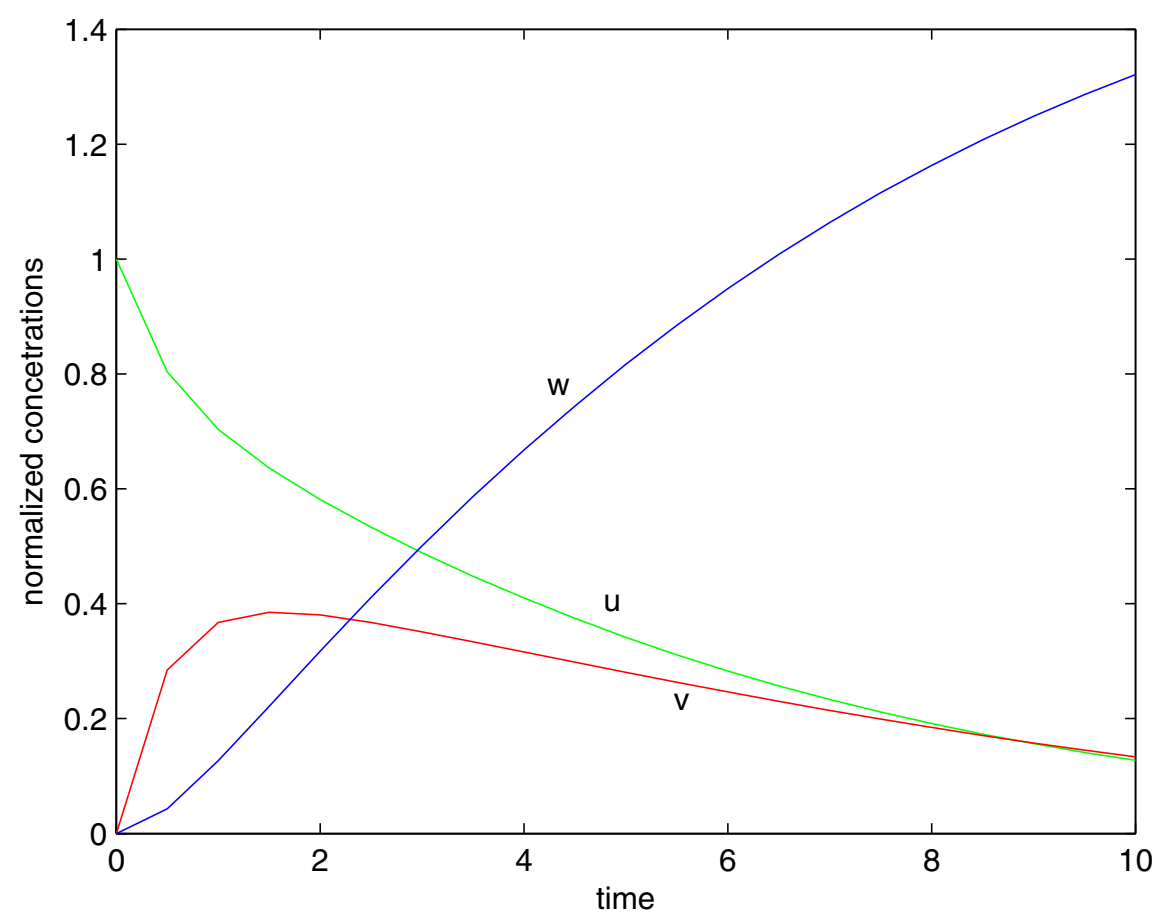

Figure 3: Profile of the normalized concentrations of the substrate $u$, enzyme-substrate complex $v$, and product $w$ for $k_{3}=1, \lambda=0.5, \varepsilon=0.6$, and $\alpha_{i}=1, i=1,2,3$; DTM solution for $\Delta t=h^{\prime}=0.5, M=20, N^{\prime}=2$.

for discrete version of model (24) having time step $h$ and $\alpha_{i}=1$ and MSDTM and fourth-order Runge-Kutta method for continuous version of model (24) having time step $h \rightarrow 0+$ and $\alpha_{i}=1$. All three methods is giving the same solution for corresponding model with model parameters $k_{3}, \epsilon$, and $\lambda$ that are shown in caption of corresponding figure. Basic parameters for both cases are: $\Delta t=0.1, h=0.01, M=100, N=10$.

Next, for the model (24) discrete and continuous version with $\alpha_{i}=1$, the influence of the increased time step $h$ on the kinetics, is considered (Figures 3,4 and 5 ). It can be concluded that the MSDTM is more stable numerical algorithm then the MSDETM.

If numerical solutions presented in Figures 1 and 2, are taken as referent, then based on Figures 4 and 5, it can be concluded that DETM is significantly less accurate than DTM. 


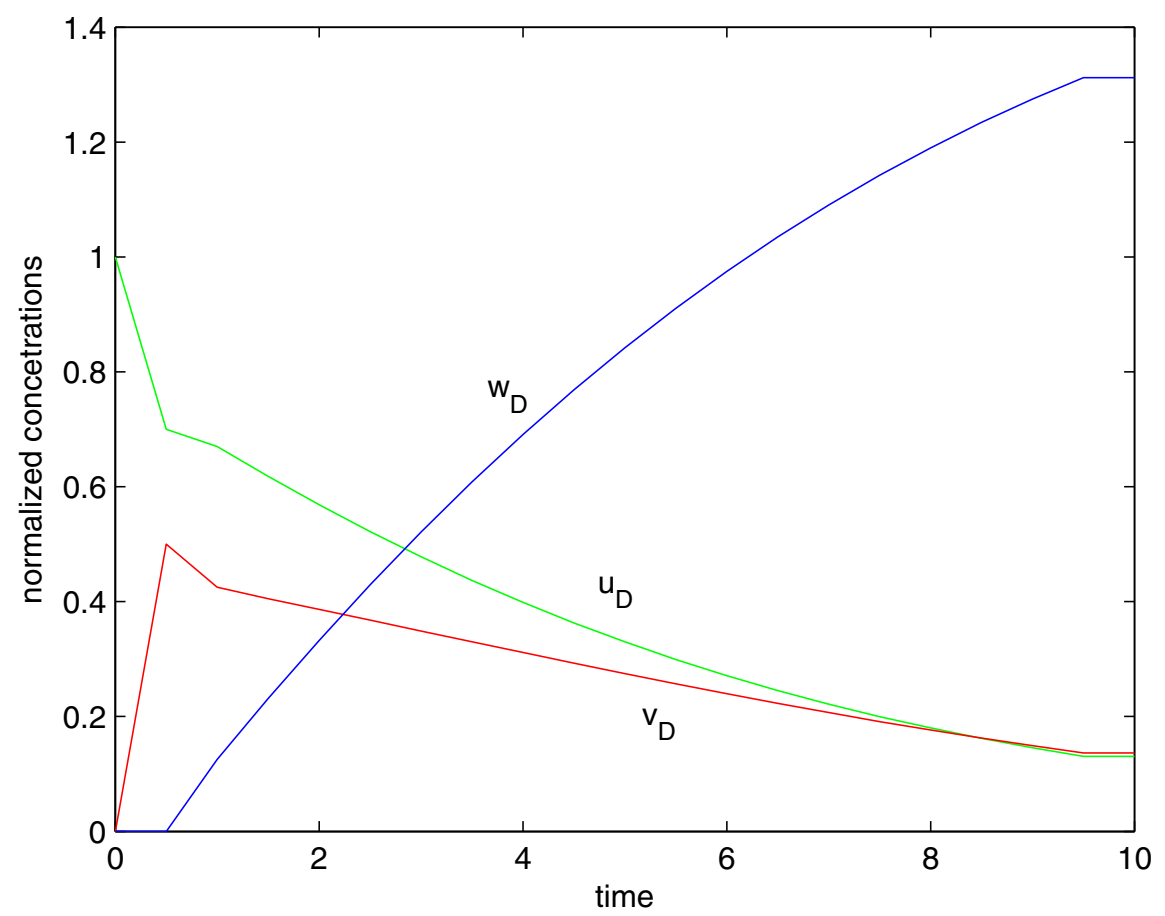

Figure 4: Profile of the normalized concentrations of the substrate $u_{D}$, enzyme-substrate complex $v_{D}$, and product $w_{D}$ for $k_{3}=1, \lambda=0.5, \varepsilon=0.6$, and $\alpha_{i}=1, i=1,2,3$; DETM solution for $\Delta t=0.5, h^{\prime}=0.5, M=20, N^{\prime}=2$. 


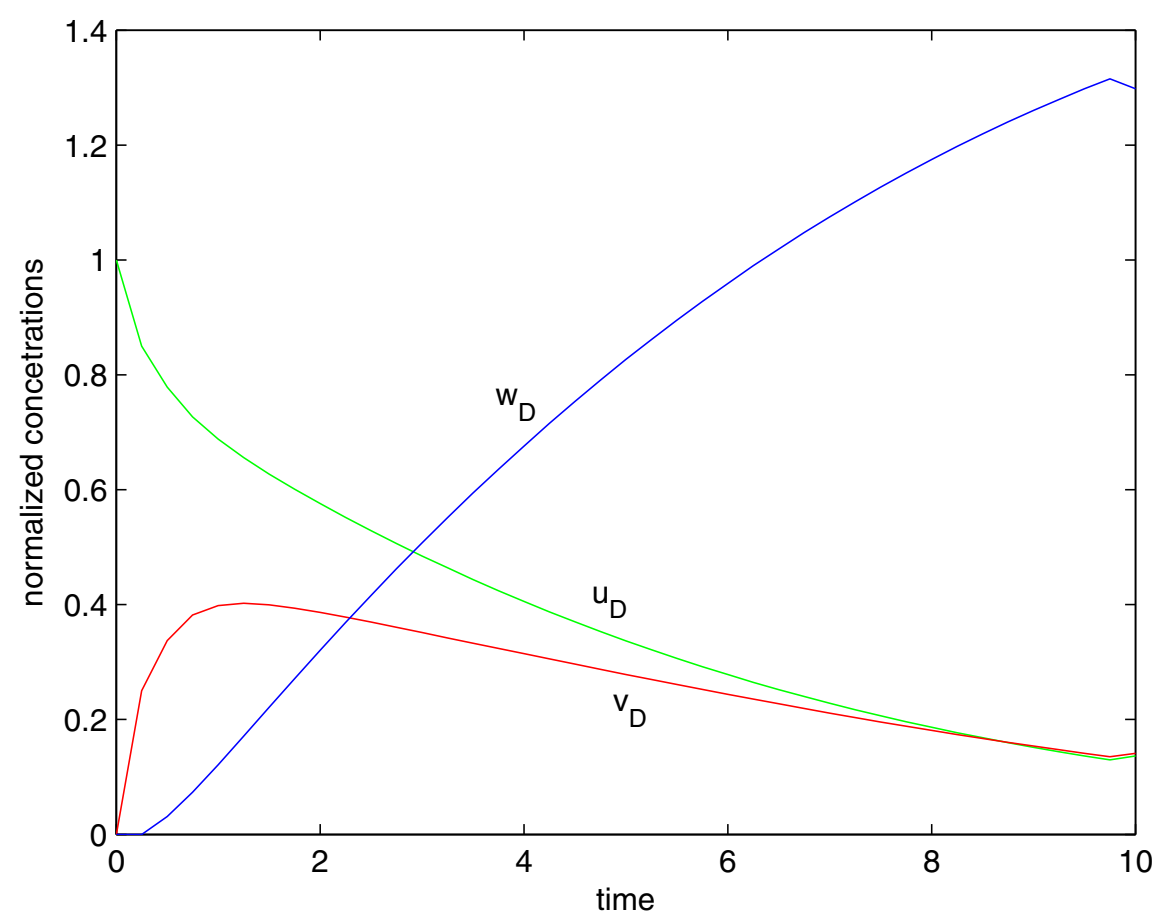

Figure 5: Profile of the normalized concentrations of the substrate $u_{D}$, enzyme-substrate complex $v_{D}$, and product $w_{D}$ for $k_{3}=1, \lambda=0.5, \varepsilon=0.6$, and $\alpha_{i}=1, i=1,2,3$; MSDETM solution for $\Delta t=0.5, h^{\prime}=0.25, M=20, N^{\prime}=4$. 


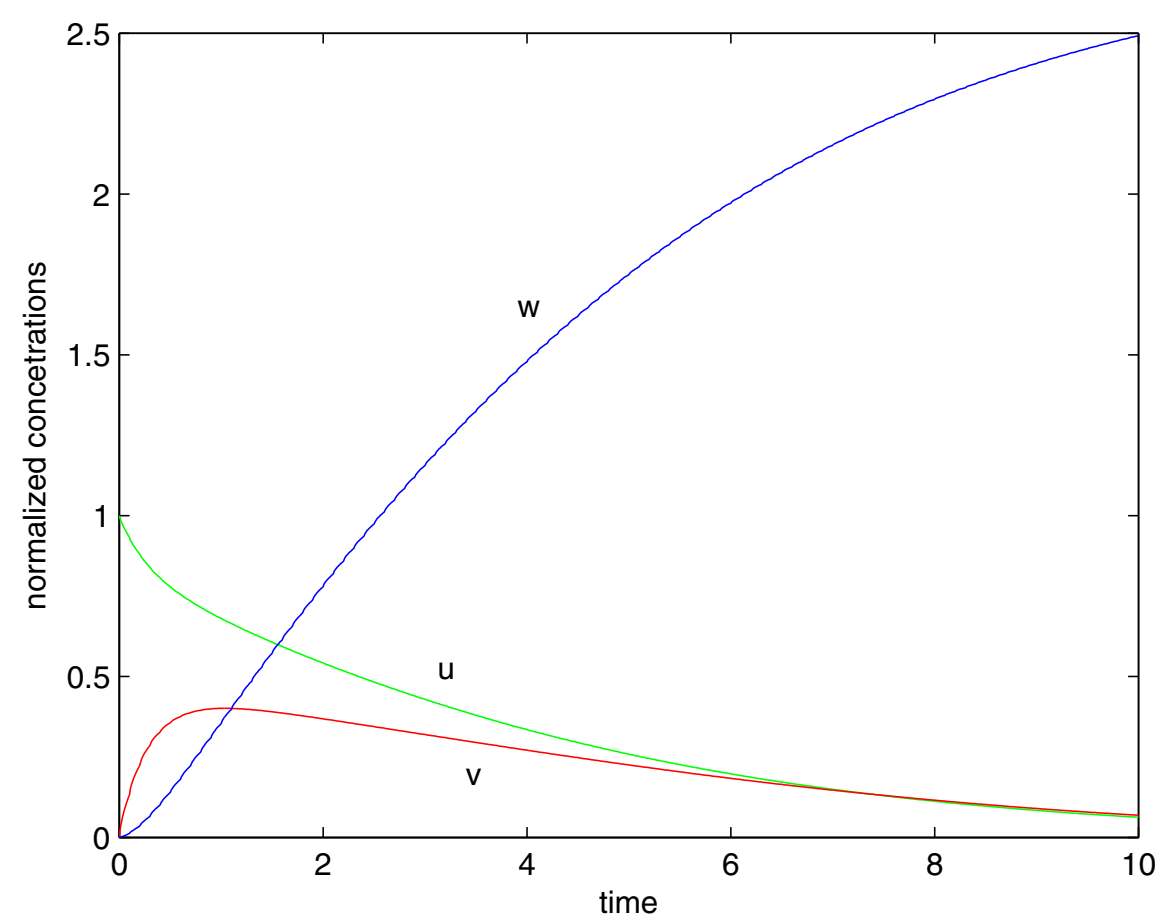

Figure 6: Profile of the normalized concentrations of the substrate $u_{D}$, enzyme-substrate complex $v_{D}$, and product $w_{D}$ for $k_{3}=1, \lambda=0.5, \varepsilon=0.6$, and $\alpha_{1}=0.9, \alpha_{2}=0.8$, $\alpha_{3}=0.7$; MSDGTM solution for $\Delta t=0.1, h=0.01, M=100, N=10$. 


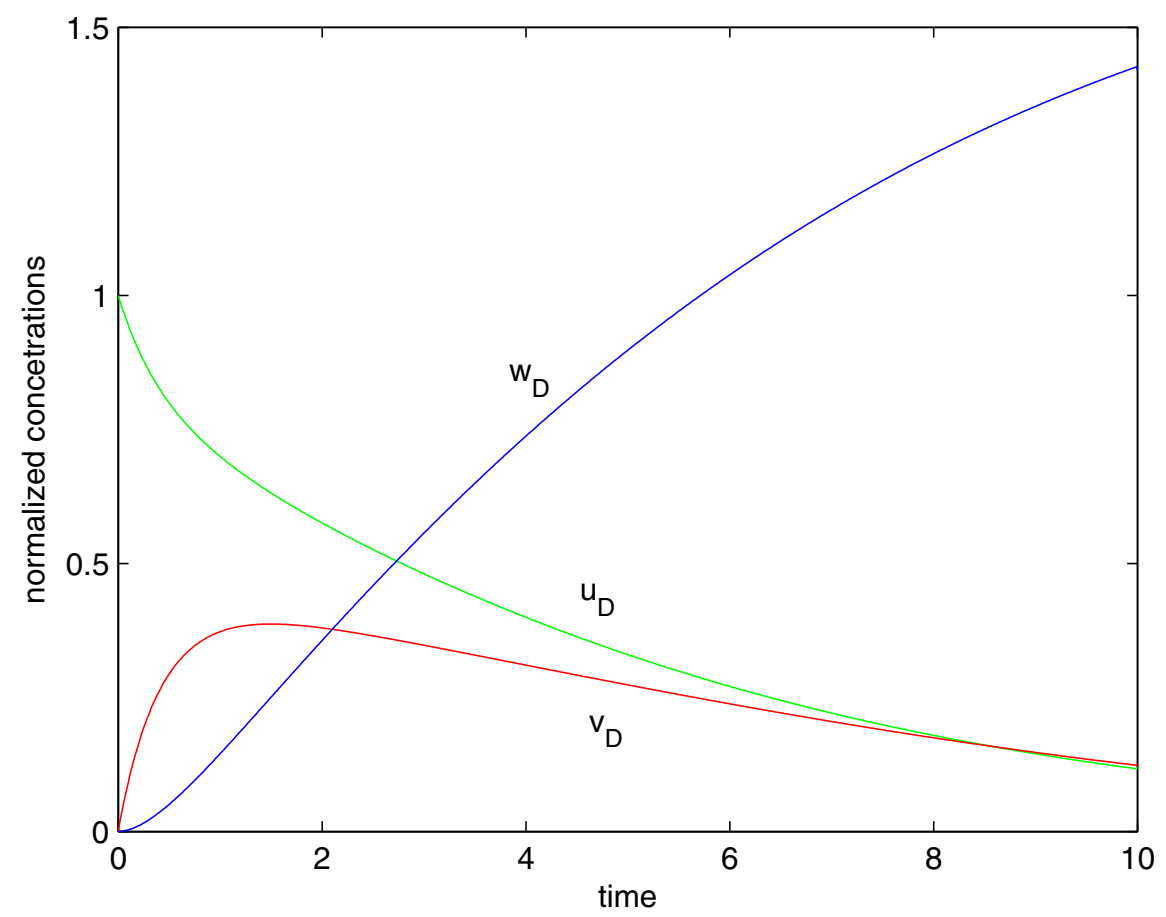

Figure 7: Profile of the normalized concentrations of the substrate $u$, enzyme-substrate complex $v$, and product $w$ for $k_{3}=1, \lambda=0.5, \varepsilon=0.6$, and $\alpha_{1}=0.9, \alpha_{2}=0.8, \alpha_{3}=0.7$; MSGDETM solution for $\Delta t=0.1, h=0.01, M=100, N=10$.

Figure 6 presents graphically MSDGTM solution for model (24) with $\alpha_{1}=0.9, \alpha_{2}=0.8, \alpha_{3}=0.7$ and it does not significantly differ from the case when parameter values are $\varepsilon=0.5, k_{3}=2$ and $\lambda=1.0$. This means that the influence of the parameters $\alpha_{i}$ is dominant in this case.

Compared to the case previously presented in Figure 6, graphs in Figures 7 and 8 are very different, because all the parameters have equal influence on the flow of enzymatic reaction.

In Figure 9, when $h^{\prime}=0.5>h=0.01$, graph is identical to graph in the Figure 6. Therefore: (1) results obtained with MSGDTM and GTM are the same and (2) the influence of the parameters $\alpha_{i}$ in this case is dominant too.

Figures 10 and 7, show that GDETM and MSGDETM solutions are very different. The product creation velocity is significantly lower than the formation of the complex enzyme - substrate (please refer to Eqs. (24) and their 


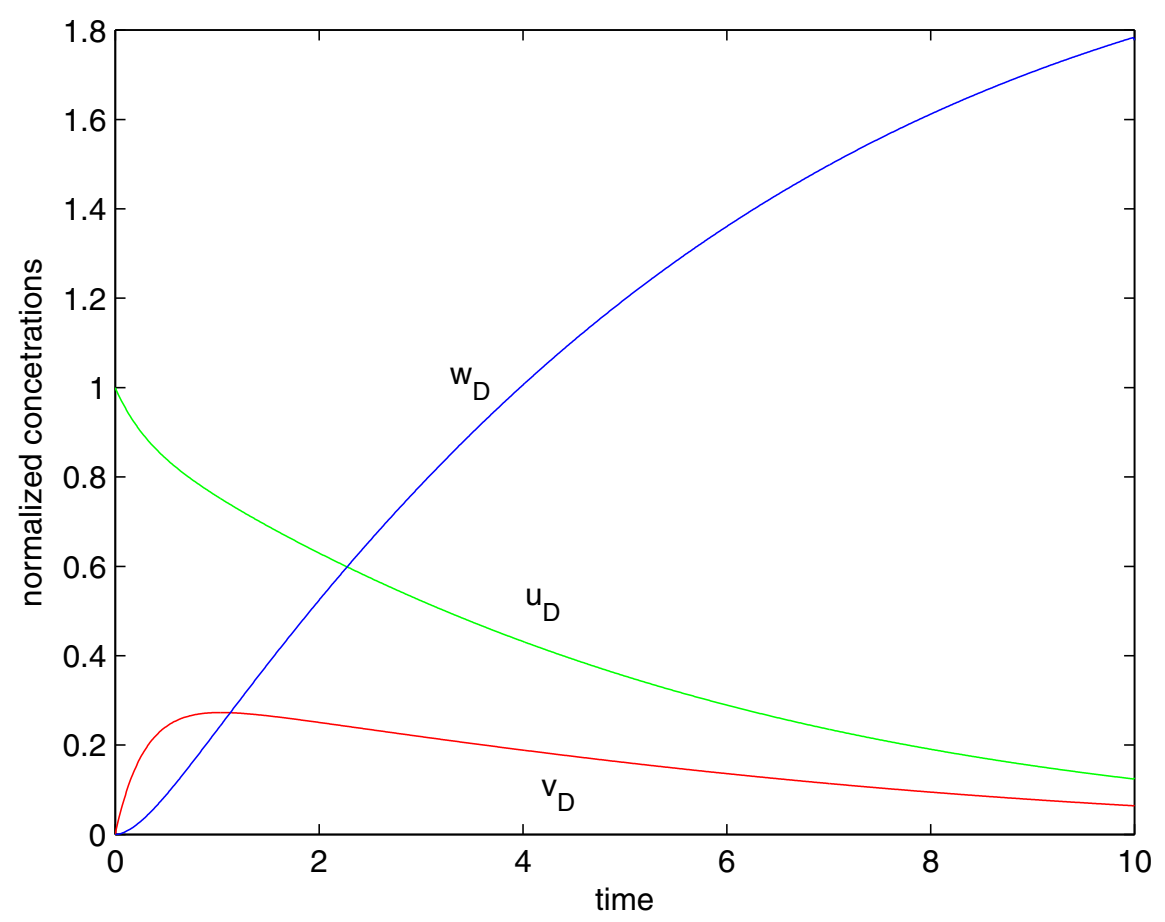

Figure 8: Profile of the normalized concentrations of the substrate $u_{D}$, enzyme-substrate complex $v_{D}$, and product $w_{D}$ for $k_{3}=2, \lambda=1.0, \varepsilon=0.5$, and $\alpha_{1}=0.9, \alpha_{2}=0.8$, $\alpha_{3}=0.7$; MSGDETM solution for $\Delta t=0.1, h=0.01, M=100, N=10$. 


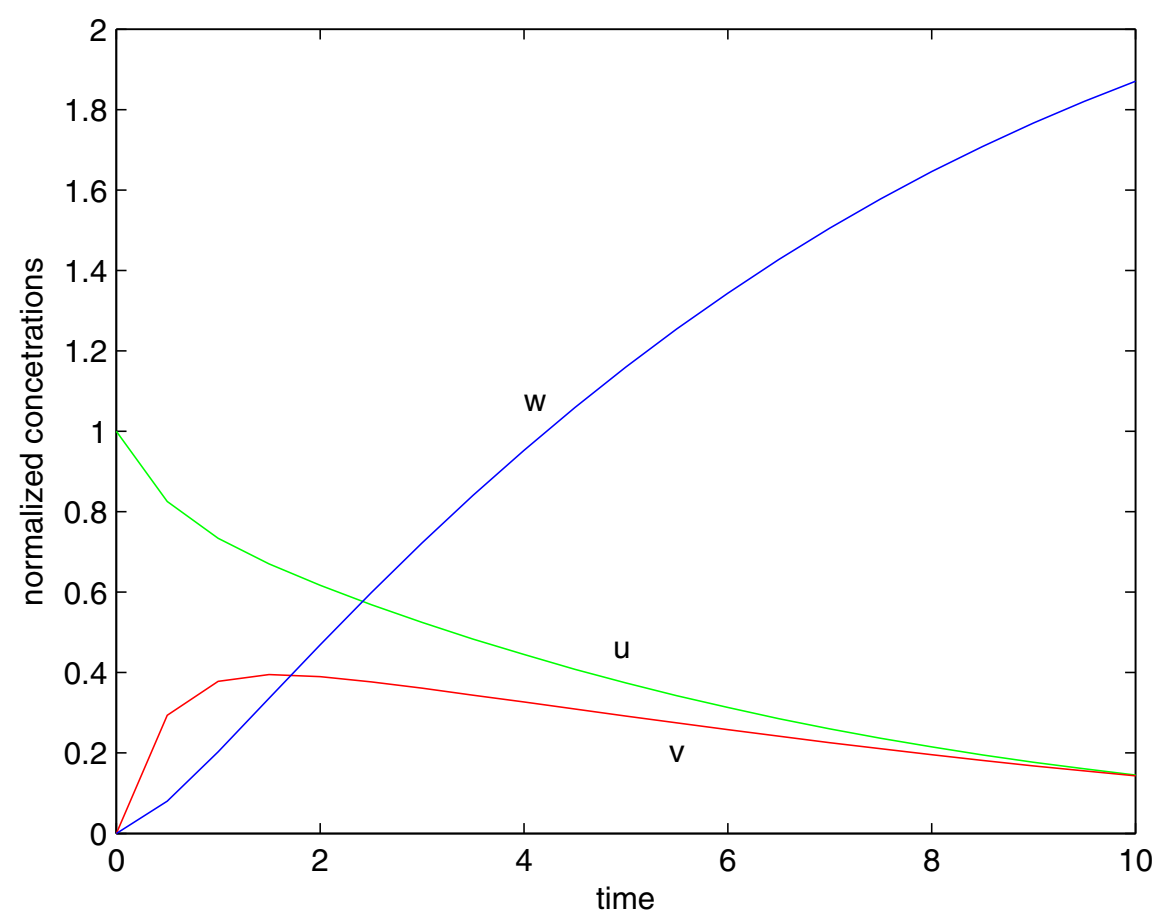

Figure 9: Profile of the normalized concentrations of the substrate $u_{D}$, enzyme-substrate complex $v_{D}$, and product $w_{D}$ for $k_{3}=1, \lambda=0.5, \varepsilon=0.5$, and $\alpha_{1}=0.9, \alpha_{2}=0.8$, $\alpha_{3}=0.7$; GDTM solution for $\Delta t=h^{\prime}=0.5, M=20, N^{\prime}=2$. 


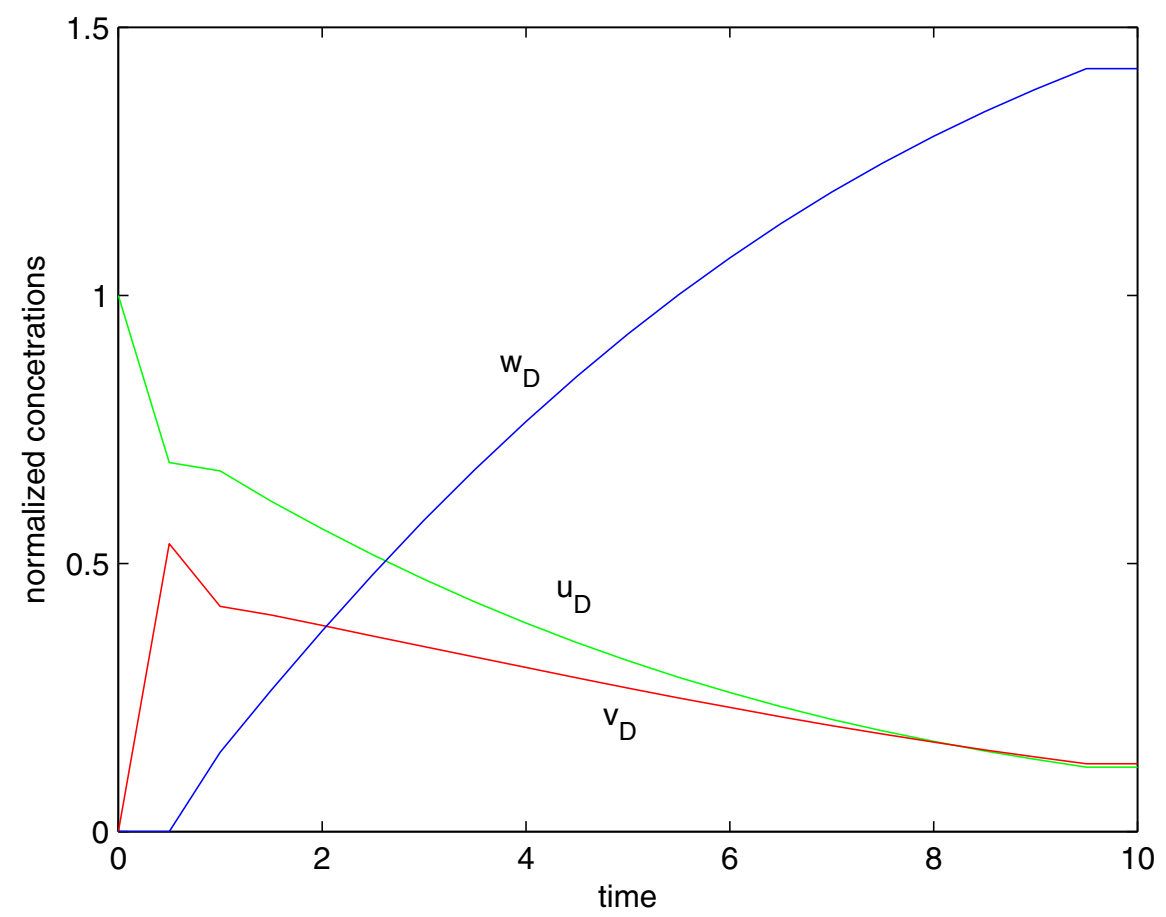

Figure 10: Profile of the normalized concentrations of the substrate $u_{D}$, enzyme-substrate complex $v_{D}$, and product $w_{D}$ for $k_{3}=1, \lambda=0.5, \varepsilon=0.6$, and $\alpha_{1}=0.9, \alpha_{2}=0.8$, $\alpha_{3}=0.7$; GDETM solution for $\Delta t=h^{\prime}=0.5, M=20, N^{\prime}=2$.

initial conditions), as the value of the time step $h$ influences the nature of the solutions of the above mentioned system, and approximation represented in Figure 10 exhibits corner points.

Graphics in Figure 8 and Figure $11\left(h^{\prime}=h\right.$ and $h^{\prime}=25 h$, respectively), reflect the high impact of changes of steps $h^{\prime}$ to the concentrations time dependence.

The main conclusions for the MSGDETM and MSGTM numerical experiments regard to the fractional discrete and continuous Michaelis-Menten model, are: (1) The time scale of the biophysical (biochemical) processes, parameter $h$, in addition to the memory effect, could be important characteristic of specific system; (2) Degrees of fractional differences are the parameters that significantly influence the rate of the reactions (24).

At the end, it is reasonable to consider the extraction of parameters in the 


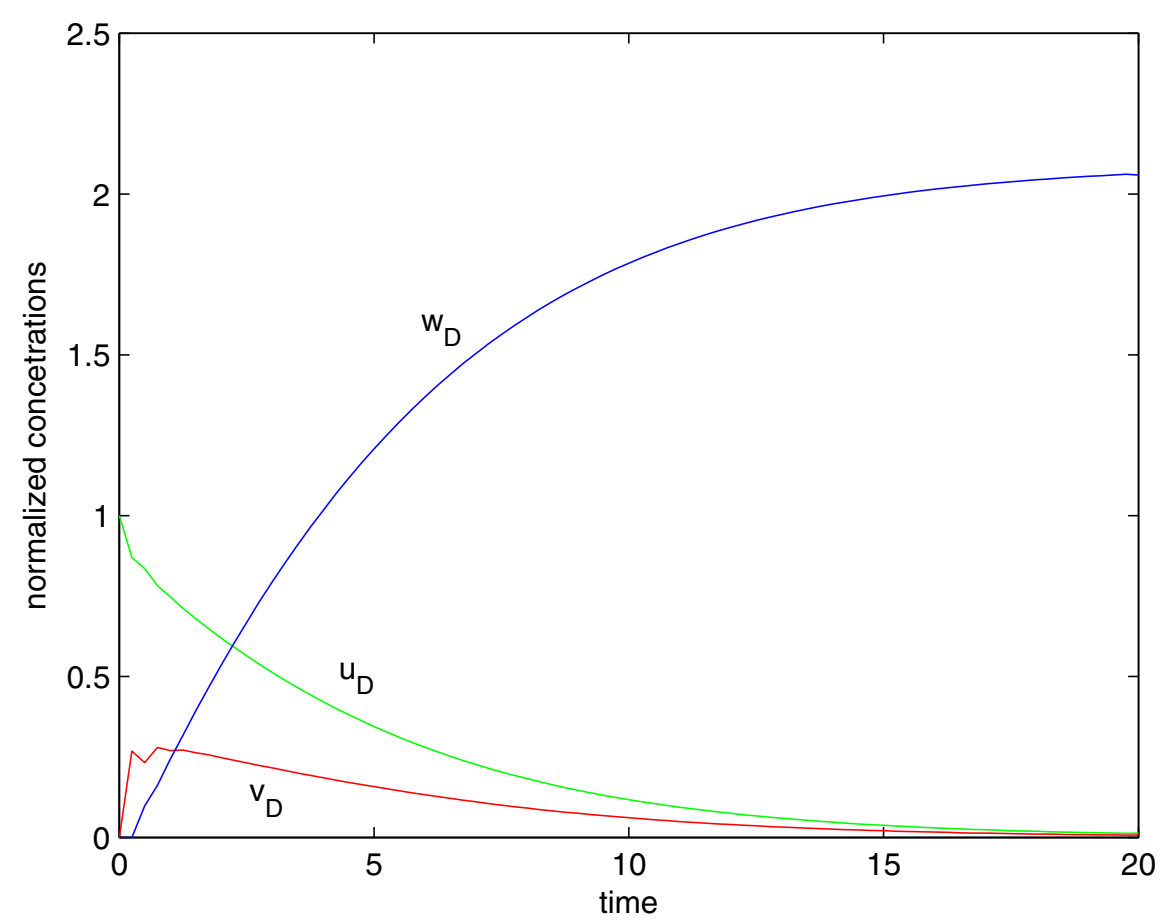

Figure 11: Profile of the normalized concentrations of the substrate $u_{D}$, enzyme-substrate complex $v_{D}$, and product $w_{D}$ for $k_{3}=2, \lambda=1.0, \varepsilon=0.5$, and $\alpha_{1}=0.9, \alpha_{2}=0.8$, $\alpha_{3}=0.7$; MSGDETM solution for $\Delta t=0.5, h^{\prime}=0.25, M=40, N^{\prime}=4$. 
case of experiments, as is done in reference [35]. The applied MATLAB's lsqnonlin procedure, where the minimized appropriate function: (1) calculate the enzyme kinetics model solutions (using ode23 or ode23s solvers) for a given set of rate constants and initial conditions (optimization procedure parameters), (2) evaluates the solutions for the time points corresponding to the observations and (3) subtracts the experimental data. Initial parameters are obtained using the Manipulate and NDSolve functions of CAS Mathemat$i c a$ in order to get a rough fit of the experimental data. Extracted parameters are: $k_{1}, k_{-1}, k_{2}, s_{0}$ and $e_{0}$. In order to solve a similar problem within the framework of fractional difference Michaelis-Menten model, it is necessary to generalize ode23 or ode23s solvers, i.e. formulate stable fractional difference (or differential) Runge-Kutta method in Caputo sense (in literature, this method is known for Riemann-Liouville fractional derivatives [42]). On the other hand, as mentioned above [19], MSDTM is comparable to ordinary Runge-Kutta fourth order method, and by using MSGDTM, the approximate solutions are highly accurate and valid for a long time. Consequently, in the case where is no fractional difference (i.e. differential) Runge-Kutta method in Caputo sense, the basis of this and future investigations, exists and should be MSGDETM.

\section{Conclusions}

The main contribution of this paper is generalized model of MichaelisMenten model of enzyme kinetics, which overcomes the shortcomings of the original model given in reference [14]. In particular, as was mentioned in the introduction, the assumption of irreversibility of the Michaelis-Menten reaction is acceptable under two conditions: when the Gibbs free energy released in the reaction is very large and when the substrate concentration is much higher than that of product, but when this is not a case, the reaction cannot be presented by standard Michaelis-Menten model [14]. In this respect, the upgraded, generalized, Michaelis-Menten model is proposed and consequently a new algorithm for it solution was needed. Therefore, the formulation of new fractional calculus based on TSC as well as its application in form of MSGDETM to obtain numerical solutions of a generalized time fractional Michaelis - Menten model of enzyme kinetics is proposed. The multi-step GDETM as the new method for solving difference equations was precisely defined and it can be considered as a base for direct experimental verification of the appropriate models under different conditions. As it can 
be seen from the Figures 6 and 7, continuous and discrete Caputo fractional models have completely different numerical solutions of enzyme kinetics for sufficiently large values of the time step $h$ (this parameter in this research is taken as material constant, which as such, has not been considered yet). It is obvious that the proposed model is giving much better results than convectional approaches presented in literature. Figures 1 and 2 show that they have, on the contrary, in the simplest case, approximately the same solutions. That represents one of the basic ideas for the formulation of TSC. The next step in the research is to discuss concrete experimental data and models.

\section{Acknowledgment}

The authors gratefully acknowledge dr Zoran Kkic whose thoughtful and constructive comments improved the overall quality of this publication.

\section{References}

[1] Voit E O. Biochemical Systems Theory: A Review. Isrn Biomath 2013;897658:53.

[2] Keener J, Sneyd J. Mathematical Physiology I: Cellular Physiology. Springer Science+Business Media, LLC, NeW York, 2009.

[3] Magin R L. Fractional Calculus in Bioengineering. Begell House, Redding, Càlif, USA, 2006.

[4] Xia Y, Han M. Multiple periodic solutions of a ratio-dependent predatorprey model. Chaos Solitons Fract 2009;39:1100-1108.

[5] Yang, J, Zhao L. Bifurcation analysis and chaos control of the modified Chua's circuit system. Chaos Solitons Fract 2015;77:332-339.

[6] Bohner M, Peterson A. Dynamic equations on time scales. Birkhäuser, Boston, 2001.

[7] Bohner M, Peterson A, Eds. Advances in Dynamic Equations on Time Scales. Birkhäuser, Boston, 2003.

[8] Atici F M, Şengül S. Modeling with fractional difference equations. J Math Anal Appl 2010;369(1):1-9. 
[9] Savageau M A. Michaelis-Menten mechanism reconsidered: implications of fractal kinetics. J Theor Biol 1995;176(1):115-124.

[10] Schnell S, Mendoza C. Closed form solution for time-dependent enzyme kinetics. J Theor Biol 1997;187(2):207-212.

[11] Meena A, Eswari A, Rajendran L. Mathematical modelling of enzyme kinetics reaction mechanisms and analytical solutions of non-linear reaction equations. J Math Chem 2010;48(2):179186.

[12] Goh S M, Noorani S M, Hashim I. Introducing variational iteration method to a biochemical reaction model. Nonlinear AnalReal 2010;11(4):2264-2272.

[13] Hashim I, Chowdhury M S H, Mawa S. On multistage homotopy-perturbation method applied to nonlinear biochemical reaction model. Chaos Solitons Fract 2008;36(4):823-827.

[14] Alawneh A. Application of the Multistep Generalized Differential Transform Method to Solve a Time-Fractional Enzyme Kinetics. Discrete Dyn Nat Soc 2013;592938:7.

[15] Chen CK, Ho SH. Application of differential transformation to eigenvalue problems. Appl Math Comput 1996;79:173-188.

[16] Chen CK, Ho SH. Solving partial differential equation by differential transformation. Appl Math Comput 1999;106:171-179.

[17] Odibat Z, Momani S, Ertürk V S. Generalized differential transform method: application to differential equations of fractional order. Appl Math Comput 2008;197(2):467-477.

[18] Ertürk V S, Odibat Z M, Momani S. An approximate solution of a fractional order differential equation model of human $\mathrm{T}$ cell lymphotropic virus I (HTLV-I) infection of $C D 4^{+}$T-cells. Comput Math Appl 2011;62:996-1002.

[19] Odibat M Z, Bertelle C, Aziz-Alaoui M A, Duchamp G H E. A multi-step differential transform method and application to nonchaotic or chaotic systems. Comput Math Appl 2010;59(4):14621472 . 
[20] Momani S, Odibat Z. A novel method for nonlinear fractional partial differential equations: Combination of DTM and generalized Taylor's formula. J Comput Appl Math 2008;220:85-95.

[21] Odibat Z M, Shawagfeh N T. Generalized Taylors formula. Appl Math Comput 2007;186:286-293.

[22] Gatenby R, Vincent T. An Evolutionary Model Of Carcinogenesis. Cancer Res 2003;63:6212-6220.

[23] Deutsch A, Brusch L, Byrne H, de Vries G, Herzel H, Eds. Mathematical Modeling of Biological Systems, Volume I. Birkhäuser Boston, 2007;193-203.

[24] Rihan F. Numerical Modeling of Fractional-Order Biological Systems. Abstr Appl Anal 2013;816803:11

[25] Borges F, Iarosz K, Ren H, Batista A, Baptista M, Viana R, Lopes S, Grebogi C. Model for tumour growth with treatment by continuous or pulsed chemotherapy. Biosystems 2014; 114:4348

[26] Piotrowska M J. An immune system-tumour interactions model with discrete time delay: model analysis and validation, Communications in Nonlinear Science and Numerical Simulation 2015; doi: 10.1016/j.cnsns.2015.10.022.

[27] Valle P A, Starkov K E, Coria L N. Global stability and tumor clearance conditions for a cancer chemotherapy system, Communications in Nonlinear Science and Numerical Simulation 2016; doi: 10.1016/j.cnsns.2016.04.025.

[28] Zheng Q, Shen J. Dynamics and pattern formation in a cancer network with diffusion, Communications in Nonlinear Science and Numerical Simulation 27 2015; 93109.

[29] Abdeljawad T. On Riemann and Caputo fractional differences. Comput Math Appl 2011;62:1602-1611.

[30] Čermák J, Nechvátal L. On $(q, h)$-analogue of fractional calculus. J Nonlinear Math Phys 2010;17(1):51-68. 
[31] Rahmat M R S, Noorani M S M. Caputo type fractional difference operator and its application on discrete time scales. Advances in Difference Equations 2015;2015:160.

[32] Ortigueira M, Coito F. From differences to derivatives. Fract Calc Appl Anal 2004:7(4):459-471.

[33] Podlubny I, Skovranek T, Vinagre Jara BM, Petras I, Verbitsky V, Chen YQ. Matrix approach to discrete fractional calculus III: non-equidistant grids, variable step length and distributed orders. Phil Trans R Soc A 371 2013; 20120153.

[34] Kostrobij P, Tokarchuk R, Tokarchuk M, Markiv B. Zubarev nonequilibrium statistical operator method in Renyi statistics. Reaction-diffusion processes, Condens Matter Phys 2014; 17(3):33005:19.

[35] Dimitrov S, Velikova G, Beschkov V, Markov S. On the Numerical Computation of Enzyme Kinetic Parameters. Biomath Communications 2014; $1: 2$.

[36] Kilbas A A, Srivastava H M, Trujillo J J. Theory and Applications of Fractional Differential Equations. Elsevier, Amsterdam, The Netherlands, 2006.

[37] Ferreira R A C, Torres D F M. Fractional $h$-difference equations arising from the calculus of variations. Appl Anal Discrete Math 2011;5:110-121.

[38] Atici F M, and Eloe P. W. A Transform method in discrete fractional calculus. IJDE 2007;2(2):165-176.

[39] Cermák J, Kisela T, Nechvátal L. Discrete Mittag-Leffler functions in linear fractional difference equations. Abstr Appl Anal 2011;565067:22.

[40] Samko S G, Kilbas A A, Marichev O I. Fractional Integrals and Derivatives, Theory and Applications. Gordon and Breach Science Publishers, Yverdon, Switzerland, 1993. 
[41] Tsallis C. Nonextensive statistics: Theoretical, experimental and computational evidences and connections. Braz J Phys 1999; 29(1).

[42] Cao X, Li Y. Fractional Runge-Kutta Methods for Nonlinear Fractional Differential Equation. JNSA 2011; 2(3-4):189-194.

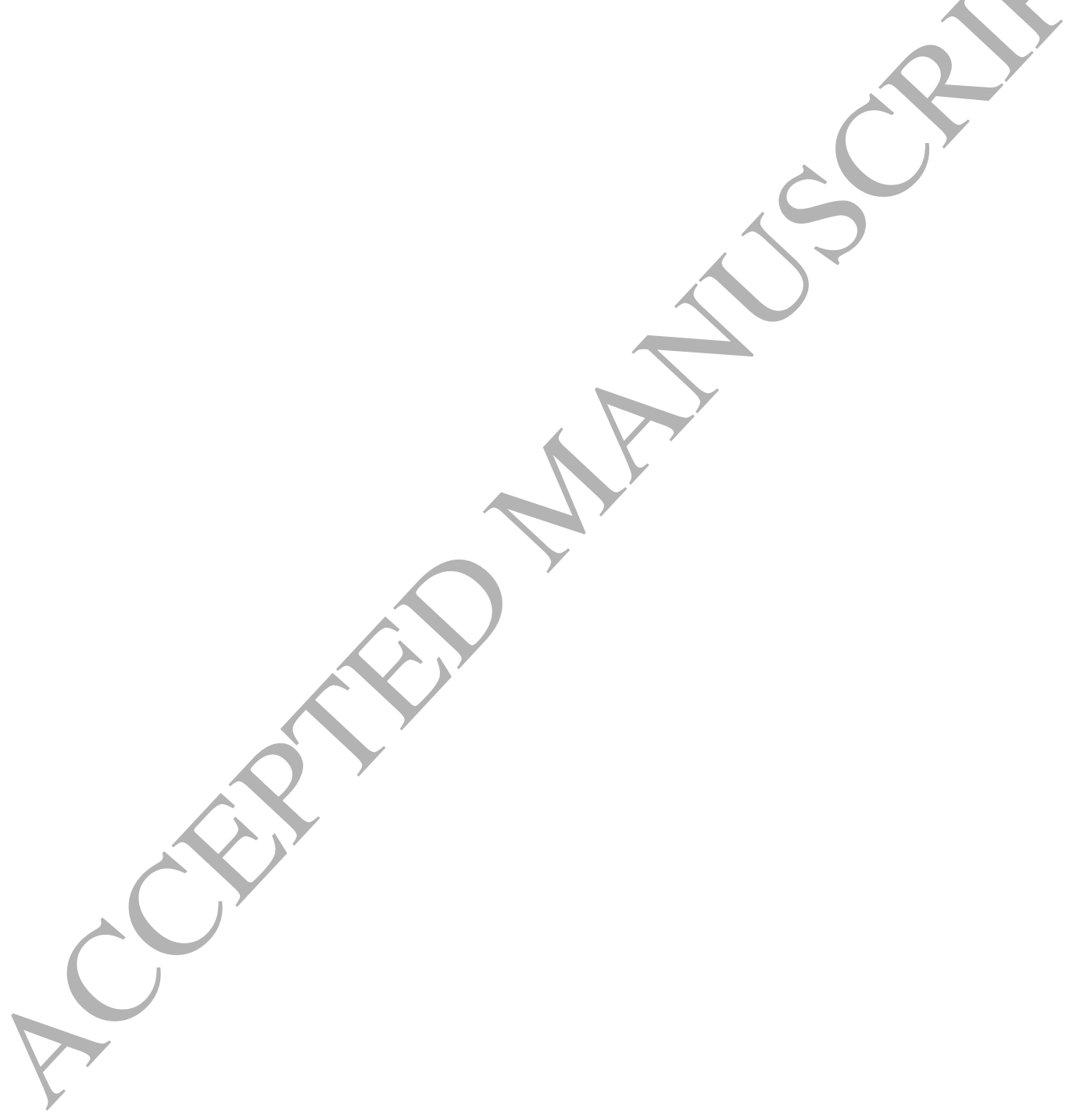

